\title{
Zur Semantik der Konjunktion als. Paradigmatische und syntagmatische Aspekte ${ }^{*}$ \\ Hardarik Blühdorn (Mannheim)
}

\begin{abstract}
This paper develops a theoretical model for the semantics of connectives, following central ideas of Reichenbachian tense semantics.

In a first step, the terminological and conceptual framework is presented and illustrated with German $d a$. The meaning of a connective is modeled as a fourplace-relation between the situated object $\mathrm{E}$, a reference object $\mathrm{R}$, a discourse anchor $\mathrm{S}$ and the speaker $\mathrm{O}$. The relata can belong to one of four different classes of entities: physical object, event, proposition or act. Correspondingly, the relations are divided into four cognitive domains: space, time, alethics/epistemics, and deontics. In each domain, relations can be treated under three different perspectives: situation, condition or causation. A crossclassification of relational domains and perspectives provides a typology of connectives which is more consistent than the ones available in traditional grammar.

In the second part of the article, the analytic apparatus is refined, using German so as the main example. Following Roman Jakobson, a distinction is made between contiguity and similarity relations. Contiguity relations are typically encoded by functional categories, whereas similarity relations are encoded by lexical categories. However, there are a few connectives like so which encode similarity relations. A structural isomorphism between similarity and contiguity relations makes it possible to reinterpret so in certain contexts as an indicator of contiguity. In these cases, so is semantically weakened, particularly in relation to its definiteness. The model is extended to also, from which als descends etymologically.
\end{abstract}

The third part of the article contains the semantic characterization of als in its variants as an intransitive and transitive connective. Als is described paradigmatically, in terms of the semantic oppositions that distinguish it from $d a$, so, wie and wenn. Like so, it originally encodes similarity relations, but in present day German its use has been extended, so that it may indicate contiguity relations as well. With $d a$ and $s o$ it shares the abstract relational meaning O-S,R,E. The main difference from $d a$ is its lesser degree of definiteness; in contrast to $s o$, its use is almost exclusively temporal. Wie and wenn are indefinites, i.e. they do

\footnotetext{
* Ich danke meinen Kollegen vom Institut für Deutsche Sprache, insbesondere Gisela Zifonun, Bernd Wiese und Reinhard Fiehler, für zahlreiche wertvolle Kommentare und Anregungen.
} 
not establish a deictic backlink to the speaker and discourse context. Als indicates that the situated event temporally overlaps with a specific event of reference, whose factivity is presupposed. The reference event must be categorically predictable in the context of utterance. Als does not indicate temporal antecedence of the reference event in relation to the speech event; it only requires the identifiability of the reference event and its non-coincidence with the speech event.

In the last section, so-called "peripheral temporal clauses" are examined with respect to the syntagmatic interaction between aspectuality, intonational focus, serialization of clauses and the abstract relational meaning of als. The proposed semantic formula is shown to be capable not only of clarifying the paradigmatic structure of a subset of German connectives but also of explaining the semantic and stylistic properties of complex sentences.

\section{$1 \quad$ Vorbemerkung}

"Wir wissen nämlich nicht nur nicht, warum immerhin 'immerhin' heißt (...), sondern wir wissen auch nicht, was es bedeutet." (Weydt 1979a: 335)

Harald Weydt hat sich über die Jahre hinweg immer wieder mit Partikeln befasst, die zugleich Satzkonnektoren sind: mit aber, denn, eigentlich, doch, immerhin, jedenfalls, schließlich, wenigstens, ohnehin, eh, sowieso und anderen (cf. z. B. Weydt 1979a, 1979b, 1979c, 1983, Hentschel/Weydt 1983). Neben ihren pragmatischen Funktionen und Verwendungsweisen hat er dabei stets auch ihre Semantik ins Auge gefasst, die sich häufig, mit Bublitz (1977: 201f., 1978: 48u.ö.) zu sprechen, in Form von "Quasi-Syllogismen" ausbuchstabieren lässt: Mitverstandene oder präsupponierte Prämissen führen zu offenen oder verdeckten Schlussfolgerungen, zu denen weitere Propositionen mittels Modalpartikeln und/oder Konjunktionen in semantische Beziehungen gesetzt werden.

Die Semantik der Satzkonnektoren ist bis heute ein Gebiet geblieben, auf dem es zahllose offene Fragen gibt, so zum Verhältnis der Wortarten, zu Polysemie/Homonymie und Synonymie, zur Paradigmatizität und zur syntaktisch-semantischen Kompositionalität. Am Institut für Deutsche Sprache befasst sich eine Forschergruppe mit dieser Thematik (http://www.idsmannheim.de/gra/konnektoren/konhome.html), die immer wieder auf Arbeiten von Harald Weydt zurückgreift. Es liegt also nahe, den Jubilar mit einem Aufsatz zur Konnektorensemantik zu ehren. 


\section{Begriffliche Voraussetzungen}

Im folgenden befasse ich mich mit paradigmatischen und syntagmatischen Aspekten der Bedeutung von als. Aus den zahlreichen Verwendungsvarianten dieses Wortes wähle ich den Gebrauch als temporale Konjunktion aus (cf. Hentschel/Weydt 1990: 275), der nach Hahnemann (1999: 29) etwa 10\% der als-Vorkommen in der deutschen Gegenwartssprache ausmacht:

(1) Sie überlegte sich eben, (so gut es ging, denn sie war schläfrig und dumm von der Hitze,) ob es der Mühe werth sei aufzustehen und Gänseblümchen zu pflücken, um eine Kette damit zu machen, als plötzlich ein weißes Kaninchen mit rothen Augen dicht an ihr vorbeirannte. (Lewis Carroll, Alice's Abenteuer im Wunderland; deutsch von Antonie Zimmermann)

Für meine Untersuchung werde ich schrittweise ein begrifflich-konzeptionelles Instrumentarium entwickeln, dessen Anwendbarkeit weit über die Semantik von als hinausgeht. Vorsichtshalber möchte ich den Leser dazu einladen, an Stellen, an denen mein Ansatz auf den ersten Blick spekulativ erscheinen mag, Einwände so lange zurückzustellen, bis seine Leistungskraft in der praktischen Analyse geprüft worden ist.

Um sich der Semantik von als zu nähern, ${ }^{1}$ ist es nützlich, zunächst ein ähnliches, aber besser erforschtes Vorbild zu betrachten, nämlich $d a$ (cf. z. B. Moilanen 1979; Ehrich 1982; Redder 1990: 132-314). Zwischen als und $d a$ bestehen zahlreiche Zusammenhänge (cf. Paul 1992: 25), die durch Beispiele wie die folgenden schlaglichtartig beleuchtet werden:

(2) Und als wir drüber war'n, da sangen alle Vöglein. (Volkslied Jetzt fahr'n wir übern See) (temporal-anaphorische Wiederaufnahme von als durch $d a$ )

(3) Da (=als) sie ihre Nägel wieder in Ulrichs Arm eingrub, tat sie es vielleicht zu stark. (Musil; zit. nach Duden 1998: 405) (partielle Synonymie von $a l s$ und $d a$ )

Die temporale Konjunktion als ist seit frühneuhochdeutscher Zeit zunehmend an die Stelle von älterem temporalem $d a$ getreten (cf. Hartweg/Wegera 1989: 139). Dieser Prozess ist in der Gegenwartssprache weitgehend abgeschlossen. Daher klingen Beispiele wie (3) antiquiert.

$D a$ ist außerordentlich polysem (cf. Paul 1992: 156ff.). Es kann

- räumlich, zeitlich, epistemisch und deontisch

- realdeiktisch und textphorisch

- situierend, konditional und kausal

${ }^{1}$ Cf. dazu die materialreiche Abhandlung von Thurmair (2001: 41ff.), die allerdings, ebenso wie Hahnemann (1999), nur am Rande auf den Gebrauch von als als Temporalkonjunktion eingeht. 
verwendet werden und gehört mindestens zwei Wortarten an: Adverb (wie in (2)) und Konjunktion (wie in (3)).

An anderer Stelle (Blühdorn 2002) habe ich die Bedeutung von da mit Hilfe eines an Reichenbachs Tempussemantik (1947=1999: 273-284) orientierten Relationenmodells untersucht (cf. dazu auch Ehrich 1992; Zifonun et al. 1997: 1151ff.). Demnach zeigt $d a$ in einem Satz wie:

(4) Da liegt der Schlüssel.

an, dass sich das situierte Objekt E (der Schlüssel) in der Nähe eines Bezugsobjektes R befindet (bei realdeiktischem Gebrauch nicht erwähnt, sondern durch eine äußerungsbegleitende Geste gezeigt; cf. Moilanen 1979: 188ff.; Blühdorn 2002), das seinerseits in der Äußerungssituation auffindbar sein muss und somit als Diskursanker S dient, aber nicht der Sprecher O sein darf. Diese relationale Bedeutung kann durch die Formel O-S,R,E kodiert werden, in der der Strich für Entfernung bzw. Nicht-Identität und das Komma für Nähe bzw. Identität steht. $\mathrm{S}, \mathrm{R}$ und $\mathrm{E}$ sind ähnlich zu verstehen wie in Reichenbachs Tempusformeln (Sprechzeit, Referenzzeit, Ereigniszeit). Anders als Reichenbach verwende ich die Buchstaben-Siglen aber als Platzhalter für Objekte und ihre Funktionen innerhalb der Relation, nicht für Zeitintervalle oder Situierungen. $D a$ stellt eine Relation her, deren Relate vier Funktionen wahrnehmen:

(5) E situiertes (lokalisiertes) Objekt (der aktuelle Referent)

R Bezugsobjekt (der Ausgangspunkt für die Auffindung des situierten Objektes)

S Diskursanker (ein Objekt in der Äußerungssituation, von dem aus das Bezugsobjekt aufgesucht wird)

O Sprecher (derjenige, der den Diskursanker festlegt)

Der Diskursanker stellt bei deiktischen Relationen eine Verbindung zwischen dem Gesagten und dem Äußerungskontext her. Er kann mit dem Sprecher und/oder mit dem Bezugsobjekt zusammenfallen, aber auch von beiden verschieden sein. So zeigt etwa das Adverb hier an, dass der Sprecher als Diskursanker und zugleich als Bezugsobjekt für die Lokalisierung des Referenten (in seiner Nähe) fungiert. Dies wird durch die Formel O,S,R,E ausgedrückt. Auch dort zeigt an, dass der Sprecher zugleich Diskursanker ist, aber das Bezugsobjekt, in dessen Nähe der Referent aufgesucht werden soll, ist von ihm entfernt (O,S-R,E). Da wiederum zeigt an, dass der Sprecher nicht Diskursanker ist, dass der Diskursanker aber zugleich als Bezugsobjekt fungiert, in dessen Nähe der Referent gesucht werden soll (O-S,R,E) (cf. im einzelnen mit Beispielen Blühdorn 2002; meine Analyse weicht in entscheidenden Punkten von anderen bekannten Ansätzen, etwa Ehrich 1992, ab).

Nach übereinstimmender Meinung der Fachgelehrten referiert $d a$ in einem Beispiel wie (4) auf einen Ort (cf. Ehrich 1992: 14), das heißt, es zeigt den Ort 
an, an dem ein Objekt (hier ein Schlüssel) aufgesucht werden soll. In (4) könnten wir statt $d a$ etwas expliziter auch auf dem Tisch sagen:

(6) Auf dem Tisch liegt der Schlüssel.

In gewisser Weise verhalten sich Präpositionalphrasen bei der Beschreibung von Orten ikonischer als Adverbien. Sie lassen deutlicher erkennen, dass ein Ort eine Relation ist (cf. Frawley 1992: 251, 254ff.), genauer gesagt, eine Relation mit einer ungesättigten Leerstelle. Diese Leerstelle nimmt das situierte Objekt auf, das sich an dem Ort befindet. Der Ort in Beispiel (6) ist auf dem Tisch. Der Tisch ist das Bezugsobjekt R. Die Präposition auf stiftet eine zweistellige Relation, deren eine Leerstelle durch $\mathrm{R}$ gesättigt wird. Die zweite Leerstelle ist für das situierte Objekt E (hier: den Schlüssel) reserviert.

Das Bezugsobjekt wird im vorliegenden Beispiel mit dem Definitartikel eingeführt (dem Tisch). Definitheit bedeutet Auffindbarkeit (z. B. in der Äußerungssituation), in der dritten Person zugleich Nicht-Identität mit dem Sprecher. Bezüglich der Definitheit des Bezugsobjektes verhalten auf dem Tisch und $d a$ sich gleich und stehen in Opposition zu Ortsangaben mit indefinitem Bezugsobjekt, etwa auf einem Tisch.

Der Unterschied zwischen auf dem Tisch und $d a$ besteht darin, dass $d a$ das Bezugsobjekt nicht explizit macht, sondern lediglich seine Auffindbarkeit anzeigt. Damit das Bezugsobjekt im Kommunikationskontext tatsächlich aufgefunden werden kann, werden Zeigegesten eingesetzt.

Syntaktisch verhalten auf dem Tisch und $d a$ sich ähnlich. Da kann auf dem Tisch substituieren. Beide Ausdrücke haben nur eine syntaktische Leerstelle, die mit einem Ausdruck für das situierte Objekt besetzt werden muss. Mit Wunderlich (1984: 82f.; cf. auch Bierwisch 1988: 3) können wir auch sagen, dass Adverbien wie $d a$ und Präpositionalphrasen wie auf dem Tisch intransitiv (syntaktisch einstellig) sind. Präpositionen wie auf sind dagegen transitiv (syntaktisch zweistellig).

Da kann nicht nur realdeiktisch, sondern auch anaphorisch (zur Wiederaufnahme zuvor in den Diskurs eingeführter Referenten als Bezugsobjekte für die Situierung weiterer Referenten) verwendet werden:

(7) Sein Blick fiel auf einen Tisch in der Ecke. Da lag der Schlüssel.

In diesem Beispiel wird der zuvor eingeführte Tisch durch $d a$ wiederaufgenommen und als Bezugsobjekt für die Lokalisierung des Schlüssels genutzt. $D a$ bringt als eigenen semantischen Beitrag die Relation der Nähe zwischen dem zu lokalisierenden Objekt E und dem Bezugsobjekt R ins Spiel. Bei anaphorischem Gebrauch wird, anders als bei realdeiktischem Gebrauch, das Bezugsobjekt im vorausgehenden Text erwähnt. Dann entfällt die Notwendigkeit von Zeigegesten (cf. Blühdorn 2002). 
Neben anaphorischem ist kataphorischer Gebrauch von da möglich:

(8) Der Schlüssel lag da auf dem Tisch.

Ohne Realdeixis ist in einem Satz wie (8) möglicherweise zunächst kein Bezugsobjekt für $d a$ identifizierbar. Im Anschluss an $d a$ wird aber mit auf dem Tisch ein Bezugsobjekt nachgeliefert und damit die Interpretierbarkeit sichergestellt.

In den bisher diskutierten Beispielen zeigt $d a$ Raumrelationen an. Daneben kann es auch zur Anzeige von Zeitrelationen verwendet werden:

(9) Ich sagte, dass es Jonas gewesen sei. Da sagte er: "Wenn es Jonas war, dann ist nichts zu machen."

Raumrelationen bestehen zwischen physischen Gegenständen, also zwischen Objekten, die wir mit Lyons (cf. 1977: 442) als Entitäten erster Ordnung bezeichnen können. Zeitrelationen bestehen demgegenüber zwischen Sachverhalten (Entitäten zweiter Ordnung; cf. ebd.: 443f.). Wenn da zur Anzeige von Zeitrelationen verwendet wird, bleibt seine abstrakte relationale Bedeutung, die in der Formel O-S,R,E ausgedrückt ist, unverändert. Temporales da zeigt an, dass der zu situierende Sachverhalt in der Nähe eines Bezugssachverhaltes zu finden ist, der im Kontext identifizierbar ist, bei dem es sich aber nicht um das aktuelle Sprechereignis handelt. Temporaler Gebrauch von $d a$ ist nicht realdeiktisch, sondern nur anaphorisch möglich (realdeiktisch sind etwa bald und kürzlich), d.h. der Bezugssachverhalt muss vorerwähnt sein. In (9) wird er im ersten Satz bezeichnet: Der Sprecher sagt, dass es Jonas gewesen sei. Da im zweiten Satz nutzt diesen Sachverhalt für die Lokalisierung der Antwort in zeitlicher Nähe.

Außer räumlich und zeitlich kann $d a$ auch alethisch und deontisch verwendet werden (cf. Lyons 1977: 791ff.). Alethische (logische) Relationen bestehen zwischen Propositionen (Entitäten dritter Ordnung; cf. ebd.: 445) und betreffen deren Lokalisierung relativ zu anderen Propositionen hinsichtlich ihrer Wahrheit oder Falschheit. Ein Spezialfall der alethischen sind epistemische Relationen, die Wahrheitswerte von Propositionen deiktisch an den Sprecher binden ${ }^{2}$ (cf. ebd.: 793ff.):

(10) Wenn Japaner durch Europa reisen, dann ist ihre Zeit knapp. Man hat in Nippon nicht so viel Urlaub wie bei uns; außerdem sind da diese entsetzlich langen Anflugzeiten - da ist klar, dass man sich bei den Besichtigungen auf das Wesentliche beschränken muss.

(http://www.br-online.de/bayern2/kalenderblatt/oktober/kb19991001.html 13.09.2002)

\footnotetext{
${ }^{2}$ Seit Rauh (1983: 32) ist verschiedentlich darauf hingewiesen worden, dass auch modale Relationen deiktisch sein können (cf. etwa Diewald 1999).
} 
Epistemisches $d a$ zeigt an, dass die zu situierende Proposition in logischer Nähe zu einer Bezugsproposition steht, die im Kontext identifizierbar (gewöhnlich vorerwähnt) ist. Dabei muss es sich nicht um eine einzelne Proposition handeln, sondern Bezugsobjekt kann auch ein Propositionensystem sein. $D a$ erlaubt aber nicht, das Propositionswissen des Sprechers als Diskursanker zu wählen. Beispielsweise wäre es semantisch abweichend, ohne vorausgehende Erwähnung von Bezugspropositionen zu sagen:

(11) Da glaube ich, dass ich fliegen kann.

Soll epistemischer Sprecherbezug (Nähe zwischen O und S) angezeigt werden, so ist die Variante ohne $d a$ zu wählen:

(12) Ich glaube, dass ich fliegen kann.

Mithin folgt auch epistemisches $d a$ der Relationsformel O-S,R,E.

Deontische (ethische) Relationen bestehen zwischen Akten (wünschbaren oder bezweckbaren Ereignissen und Zuständen; cf. Lyons 1977: 823ff.; Entitäten vierter Ordnung ${ }^{3}$ ) und betreffen deren Situierung relativ zu anderen Akten hinsichtlich ihrer Erwünschtheit oder Unerwünschtheit:

(13) Heute sind wieder zehn Mitarbeiter krank. Da muss nun endlich etwas geschehen.

Die (deontische) Notwendigkeit, dass endlich etwas geschieht, wird durch $d a$ auf die zuvor beschriebene Situation bezogen, dass wieder zehn Mitarbeiter krank sind. $D a$ kann hier paraphrasiert werden durch "in dieser deontischen Situation, in dieser Konstellation von Intentionen und Zwecken". Es ist klar, dass die gegebene Beschreibung heute sind wieder zehn Mitarbeiter krank nur einen kleinen Teil dieses Bezugssystems wiedergibt. Dazu gehören noch viele weitere Faktoren, die unerwähnt bleiben, so vielleicht die Tatsache, dass die Firma dringend einen größeren Auftrag bearbeiten muss, und das Wissen um die Unmöglichkeit, dies mit reduzierter Belegschaft zustande zu bringen. Die deontische Bezugskonstellation ist in der Äußerungssituation verifizierbar (teilweise vorerwähnt, teilweise als bekannt vorausgesetzt), kann also als Diskursanker dienen, ist aber vom Sprecher unabhängig. Auch in dieser Variante entspricht $d a$ also die Relationsformel O-S,R,E.

Die folgende Tabelle gibt einen Überblick über die vier relationalen Domänen, die ich als die Hauptbereiche der menschlichen Kognition verstehen möchte:

\footnotetext{
${ }^{3}$ Entitäten vierter Ordnung kommen im Modell von Lyons nicht vor. Wenn man aber neben Raum, Zeit und Wahrheit auch den Bereich der Zwecke und der Intentionalität in die Semantik einbeziehen möchte, so ist ihre Annahme wohl unverzichtbar. Die Bezeichnung Akt übernehme ich aus Lyons' (1977: 823) Ausführungen zur deontischen Modalität.
} 


\begin{tabular}{|l|c|c|c|c|}
\hline & 1. Ordnung & 2. Ordnung & 3. Ordnung & 4. Ordnung \\
\hline $\begin{array}{l}\text { Relationale } \\
\text { Domäne }\end{array}$ & Raum & Zeit & $\begin{array}{c}\text { Alethik/ } \\
\text { Episteme }\end{array}$ & Deontik \\
\hline Entitätsklasse & $\begin{array}{c}\text { phys. Gegen- } \\
\text { stand }\end{array}$ & Sachverhalt & Proposition & Akt \\
\hline $\begin{array}{l}\text { Objekt- } \\
\text { konstitution } \\
\text { vs. Nicht- } \\
\text { Vorkommen }\end{array}$ & $\begin{array}{c}\text { Faktizität vs. } \\
\text { Nicht-Faktizität }\end{array}$ & $\begin{array}{c}\text { Wahrheit vs. } \\
\text { Falschheit }\end{array}$ & $\begin{array}{c}\text { Erwünschtheit } \\
\text { vs. Uner- } \\
\text { wünschtheit }\end{array}$ \\
\hline Situierung & Zeitpunkt & $\begin{array}{c}\text { alethische/ } \\
\text { epistemische } \\
\text { Relation }\end{array}$ & $\begin{array}{c}\text { deontische } \\
\text { Relation }\end{array}$ \\
\hline
\end{tabular}

Für jede der Domänen sind Entitäten einer bestimmten Ordnung konstitutiv. So konstituieren physische Gegenstände durch die Beziehungen, die sie untereinander eingehen, den Raum, und zwar tun sie dies kraft ihres Vorkommens. Ihre Situierung im Raum ist der Ort, an dem sie vorkommen. Sachverhalte konstituieren durch die Beziehungen, die sie untereinander eingehen, die Zeit, und zwar kraft ihrer Faktizität. Ihre Situierung ist der Zeitpunkt, zu dem sie faktisch sind. Propositionen konstituieren kraft ihrer Wahrheitsbeziehungen den Raum der Alethik bzw. Episteme. Ihre logische Situierung ist die Propositionskonstellation, in der sie wahr sind. Akte schließlich konstituieren kraft ihrer Erwünschtheitsbeziehungen den Raum der Deontik. Ihre deontische Situierung ist die Aktkonstellation, in der sie erwünscht sind.

Ich werde annehmen, dass die vier relationalen Domänen systematisch aufeinander aufbauen. Die Raumdomäne ist die Grundlage für die Zeitdomäne, beide zusammen bilden die Grundlage für die logische Domäne, und alle drei sind Grundlage für die deontische Domäne. Keine der Domänen kann aber restlos auf die übrigen zurückgeführt werden. Jede von ihnen hat ihren eigenen Geltungs- und Wirkungsbereich. So liegen deontische Objekte und Relationen außerhalb der Reichweite der Logik, logische Objekte und Relationen außerhalb der Reichweite von Zeit und Raum und zeitliche Objekte und Relationen außerhalb des Raums. Im gegenwärtigen Zusammenhang ist es unmöglich, im einzelnen auf die philosophischen und psychologischen Grundlagen dieses Modells einzugehen. Es muss der Hinweis genügen, dass es sich in der jüngeren Forschungsliteratur eingebürgert hat, die Raumdomäne als einen relativ selbstständigen, gleichwohl mit anderen Domänen interagierenden Bereich der menschlichen Kognition aufzufassen (cf. z. B. Freksa et al. 1998, 2000). In Analogie dazu werde ich die Zeitdomäne, die alethisch-epistemische Domäne

\footnotetext{
${ }^{4}$ Unter alethischen/epistemischen Relationen sind hier logische Gegensätzlichkeit, Implikation, Äquivalenz usw. zu verstehen, unter deontischen Relationen Gegensätzlichkeit, Schlüssigkeit, Gleichwertigkeit etc. in Bezug auf Zwecke und Intentionen.
} 
und die deontische Domäne behandeln und ohne weitere Rechtfertigungsversuche annehmen, dass ein wesentlicher Teil des menschlichen Wissens (und zwar der für die Konnektorensemantik entscheidende Teil) in genau diesen vier relationalen Domänen organisiert ist.

In den Beispielen (4) bis (13) wurde $d a$ durchweg situierend (lokalisierend) verwendet. Ein Referent wurde (statisch) relativ zu einem oder mehreren Bezugsobjekten verortet. Daneben sind aber auch dynamischere Verwendungen von $d a$ möglich:

(14) Du willst unbedingt alleine nach Frankreich fahren? Da muss ich wirklich lachen!

Wer dies sagt, meint höchstwahrscheinlich nicht bloß, dass das Lachen-Müssen in logischer Nähe zum nach-Frankreich-fahren-Wollen situiert ist, sondern er möchte zum Ausdruck bringen, dass das nach-Frankreich-fahren-Wollen eine Bedingung ist, die das Lachen-Müssen als Folge nach sich zieht. Statt "in dieser Situation" kann man als Paraphrase für $d a$ in solchen Beispielen "unter dieser Bedingung" verwenden.

Die konzeptuelle Unterscheidung von Situierung und Bedingung ist eine Frage der Perspektive, hängt also vom Betrachter ab. Unabhängig von der relationalen Domäne wird jedes Objekt von der äußeren Umgebung beeinflusst, in der es sich befindet. Beispielsweise erwärmt sich ein Auto, das unter einem Baum abgestellt wurde, infolge des Schattens weniger als ein Auto, das in der Sonne abgestellt wurde. Der Baum ist einerseits ein Bezugsobjekt für die Situierung des Autos, schafft andererseits Bedingungen für Eigenschaften des Autos, also für seine Beschaffenheit (allgemeiner gesprochen: sein Vorkommen) in dieser Situierung. Auch solche Bedingungsverhältnisse können durch $d a$ angezeigt werden, bzw. da kann in diesem Sinne interpretiert werden:

(15) Das Auto stand den ganzen Tag in der Sonne. Da (an dieser Stelle / unter dieser Vorkommensbedingung) hat es sich ganz schön aufgeheizt.

Ferner können Bezugsobjekte auch als Ursachen aufgefasst werden. Denken wir an den Baum als Bezugsobjekt für den Schatten. Unter statischer Perspektive ist der Schatten unter dem Baum situiert. Unter dynamischer Perspektive wird der Schatten (genauer gesagt: sein Vorkommen) vom Baum verursacht. Auch in diesem Sinne kann $d a$ verwendet werden:

(16) Auf der Gegenfahrbahn verlangsamten Hunderte von schaulustigen Autofahrern die Geschwindigkeit. Da (an dieser Stelle / unter dieser Vorkommensbedingung / dadurch verursacht) kam es in kurzer Zeit zu einem Stau von mehreren Kilometern Länge.

Situierung, Bedingung und Verursachung sind relationale Perspektiven, die in jeder der vier Domänen möglich sind. In der Raumdomäne ist das Bedingte bzw. 
Verursachte das Vorkommen oder Nicht-Vorkommen physischer Gegenstände, in der Zeitdomäne ist es die Faktizität oder Nicht-Faktizität von Sachverhalten, in der alethisch-epistemischen Domäne die Wahrheit oder Falschheit von Propositionen, in der deontischen Domäne die Erwünschtheit oder Unerwünschtheit von Akten. Die folgende Tabelle gibt eine Übersicht über die relationalen Domänen und Perspektiven:

\begin{tabular}{|l|c|c|c|c|}
\hline & $\begin{array}{c}\text { phys. Gegen- } \\
\text { stand }\end{array}$ & Sachverhalt & Proposition & Akt \\
\hline $\begin{array}{l}\text { Relationale } \\
\text { Domäne }\end{array}$ & Raum & Zeit & $\begin{array}{c}\text { Alethik/ } \\
\text { Episteme }\end{array}$ & Deontik \\
\hline Situierung & Ort & Zeitpunkt & $\begin{array}{c}\text { alethische/ } \\
\text { epistemische } \\
\text { Relation }\end{array}$ & $\begin{array}{c}\text { deontische } \\
\text { Relation }\end{array}$ \\
\hline Bedingung & $\begin{array}{c}\text { räumliche } \\
\text { Disposition } \\
\text { (Vorkommens- } \\
\text { bedingungen) }\end{array}$ & $\begin{array}{c}\text { zeitliche } \\
\text { Disposition } \\
\text { (Faktizitäts- } \\
\text { bedingungen) }\end{array}$ & $\begin{array}{c}\text { alethische/ } \\
\text { epistemische } \\
\text { Modalität } \\
\text { (Wahrheits- } \\
\text { bedingungen) }\end{array}$ & $\begin{array}{c}\text { deontische Mo- } \\
\text { dalität } \\
\text { (Erwünschtheits- } \\
\text { bedingungen) }\end{array}$ \\
\hline Verursachung & $\begin{array}{c}\text { Vorkommens- } \\
\text { verursachung }\end{array}$ & $\begin{array}{c}\text { Faktizitäts- } \\
\text { verursachung }\end{array}$ & $\begin{array}{c}\text { Wahrheits- } \\
\text { verursachung }\end{array}$ & $\begin{array}{c}\text { Erwünschtheits- } \\
\text { verursachung }\end{array}$ \\
\hline
\end{tabular}

Die Bedingungs-Zeile des Schemas steht für den Kernbereich dessen, was man üblicherweise als Modalität bezeichnet (cf. Lyons 1977: 787ff.). Dazu gehört die räumliche und zeitliche Disposition, vor allem aber die logische (alethische, epistemische) und deontische Modalität. Modale Bezugsobjekte im engeren Sinne sind Propositionen bzw. Akte, deren Wahrheits- bzw. Erwünschtheitswerte als Bedingungen für die Wahrheits- und Erwünschtheitswerte anderer (zu situierender) Propositionen bzw. Akte fungieren. Modale Nähe eines Bedingten zu seinem Bezugsobjekt (seiner Bedingung) (wiedergegeben durch die Formel: R,E) bedeutet, dass der Wahrheits- bzw. Erwünschtheitswert des Bedingten durch die Bedingung weitgehend oder vollständig festgelegt wird (hinreichende Bedingung). Modale Ferne (wiedergegeben durch: R-E) bedeutet, dass keine oder nur eine unvollständige Festlegung erfolgt (nicht-hinreichende Bedingung). Modal verwendetes $d a$ zeigt also eine hinreichende Bedingung an.

Entsprechendes gilt, mutatis mutandis, für Verursachungsrelationen (Kausalrelationen). Kausale Nähe einer Wirkung zu ihrem Bezugsobjekt (ihrer Ursache) $(\mathrm{R}, \mathrm{E})$ bedeutet, dass Vorkommen, Faktizität, Wahrheit oder Erwünschtheit der Wirkung durch das Bezugsobjekt weitgehend oder vollständig bewirkt werden (effektive Verursachung). Kausale Ferne (R-E) bedeutet, dass die Wirkung gar nicht oder nur unvollständig durch das Bezugsobjekt hervorgebracht wird (inef- 
fektive Verursachung). Kausal verwendetes $d a$ zeigt also effektive Verursachung an.

Ersichtlich liefert das vorgestellte Schema eine Systematik für die semantische Klassifikation der Satzverknüpfungen, die uns die traditionelle Grammatik (cf. Duden 1998: 788ff.) schuldig bleibt: Sachverhaltsbeschreibungen werden temporal verknüpft, Propositionen alethisch-epistemisch, Akte deontisch (z. B. final). Die traditionellen Klassen der konditionalen und kausalen Verknüpfungen sind nicht zwei weitere gleichgeordnete Gruppen, sondern kreuzen sich mit den temporalen, alethisch-epistemischen und deontischen Verknüpfungen. Sie sind nicht durch die verknüpften Glieder, sondern durch die Art der Verknüpfung definiert.

Interessanterweise können alle bis jetzt besprochenen Relationstypen durch $d a$ angezeigt werden, wobei dessen relationale Bedeutung unabhängig von Domäne und Perspektive stets O-S,R,E bleibt. Wir können diese Möglichkeiten hier aus Platzgründen nicht bis in die letzten Verästelungen hinein durchspielen. Insbesondere auf Verursachungsbeziehungen werde ich im vorliegenden Aufsatz nicht weiter eingehen.

Beispiele wie (13) und (14) lassen sich nutzen, um zu zeigen, wie intransitives (adverbiales) und transitives (konjunktionales) $d a$ zusammenhängen. Der Wechsel von der intransitiven zur transitiven Verwendung ist ein Grammatikalisierungsvorgang (cf. Lehmann 1995: 92; Di Meola 2000: 62ff., 135ff.). Er geht mit der Umstellung des Konnektors vom Hauptsatz in den Nebensatz einher. Während das Adverb da beim Ausdruck für das situierte Objekt E steht, nimmt die Konjunktion $d a$ den Ausdruck, der das Bezugsobjekt R bezeichnet, als Komplement:

(13.a) Da heute wieder zehn Mitarbeiter krank waren, muss nun endlich etwas geschehen.

(14.a) Da sie unbedingt alleine nach Frankreich fahren wollte, musste ich wirklich lachen.

Auch solche Sätze erlauben Situierungsdeutungen im Sinne von "in der gegebenen Situation, dass p", konditionale Deutungen im Sinne von "unter der gegebenen Bedingung, dass $\mathrm{p}$ " und kausale Deutungen im Sinne von "bewirkt durch die gegebene Ursache, dass p". Die Transitivierung ändert nichts an der relationalen Bedeutung von $d a$, die weiterhin durch die Formel O-S,R,E wiedergegeben wird.

Somit ist O-S,R,E die allgemeine Formel für die abstrakte relationale Grundbedeutung von $d a$. In den räumlichen, zeitlichen, epistemischen und deontischen Verwendungsvarianten, unter Situierungs-, Bedingungs- und VerursachungsPerspektive, in der Realdeixis und in der Textphorik, bei intransitivem und transitivem Gebrauch, wird sie unterschiedlich interpretiert, aber an der vierstelligen 
Relation als solcher ändert sich nichts. ${ }^{5}$ Das was in Wörterbüchern als die Polysemie von $d a$ beschrieben wird, etwa räumliches $d a_{1}$, zeitliches $d a_{2}$, modales $d a_{3}$, kausales $d a_{4}$ (cf. Duden 2001: 345f.), ist nichts anderes als die Anwendung der abstrakten relationalen Grundbedeutung auf unterschiedliche Verknüpfungsglieder bzw. Ergebnis unterschiedlicher perspektivischer Deutung der Relation.

\section{$3 \quad$ Kontiguität und Similarität}

Bevor wir zur semantischen Beschreibung von als kommen, ist es sinnvoll, in einem zweiten Schritt die Semantik von so zu betrachten (cf. Ehlich 1987; Burkhardt 1987; Paul 1992: 805ff.), von dem als etymologisch abstammt (cf. Kluge 1975: 16).

$D a$ und so verhalten sich syntaktisch und semantisch auffällig analog und könnten ohne weiteres als Synonyme bezeichnet werden, stünden sie nicht hinsichtlich eines entscheidenden Merkmals in Opposition. Während $d a$ nämlich auf einen Ort hinweist, weist so auf eine Eigenschaft hin (cf. Thurmair 2001: 27; Ehlich 1987: 287 u.ö. spricht von "Aspekte(n) an 'Objekten'"). Stellen wir uns einen Kriminalermittler vor, dessen Fragen am Tatort von einem Zeugen realdeiktisch (mit begleitenden Zeigegesten; cf. Ehlich 1987: 288f.) beantwortet werden:

(17.a) Wo lag der Tote? - Ungefähr da.

(17.b) Wie lag der Tote? - Ungefähr so.

Worin genau besteht der Unterschied zwischen Orten und Eigenschaften, und wichtiger noch - was ist ihnen gemeinsam? Orte sind, wie wir gesehen haben, Relationen mit einer ungesättigten Leerstelle, und zwar Angrenzungs- oder Kontiguitätsrelationen, also Relationen von der Art, auf die Roman Jakobson (cf. 1956=1971: 83ff.) das Übertragungsprinzip der Metonymie zurückführte. Entsprechendes gilt für Zeitpunkte, für Situierungen im logischen und im deontischen Raum sowie für Konstellationen von Bedingungen und Ursachen. All diese Relationstypen beruhen auf Kontiguität, also auf wechselseitiger Angrenzung von Entitäten in einer der vier relationalen Domänen.

Eigenschaften sind davon grundverschieden. Sie betreffen die Ähnlichkeit oder Similarität zwischen Entitäten, also solche Relationen wie die, mit denen Jakobson die Metapher erklärte (cf. ebd.: 90ff.). Eigenschaften und Orte bereiten den Philosophen gleichermaßen klassische Definitionsprobleme (cf. Lyons 1977:

\footnotetext{
${ }^{5}$ Man muss darauf hinweisen, dass die hohe Bedeutungsstabilität von $d a$ keineswegs eine Selbstverständlichkeit, sondern eher ein erwähnenswerter Sonderfall ist. Wie wir noch sehen werden, ändern andere Konnektoren des Deutschen mit dem Wechsel der semantischen Domäne und/oder der Perspektive auch ihre abstrakte Relationsbedeutung.
} 
447ff., 475ff.). Bis heute besteht kaum Einigkeit darüber, was Eigenschaften, was Orte genau sein sollen (falls eine so altmodisch ontologische Sprechweise überhaupt noch angemessen erscheint). Sowohl in der philosophischen als auch in der linguistischen Literatur werden Eigenschaften, Orte und Entitäten immer wieder miteinander vermengt.

Gehen wir von Alltagsintuitionen aus. Eigenschaften werden Entitäten zugeschrieben. Sie können ganz unterschiedlicher Natur sein (cf. Ehlich 1987: 289). Zum Beispiel kann die Ausdehnung eines Objektes, also seine Größe, eine Eigenschaft sein. Aber auch die Teilnahme an einem Sachverhalt, etwa der Vater eines bestimmten Kindes zu sein, oder Licht bestimmter Wellenlänge zu reflektieren (was vom menschlichen Auge als Farbe wahrgenommen wird), ist eine Eigenschaft. Bedingungen können Eigenschaften konstituieren. Beispielsweise ist die Eigenschaft, Klavier spielen zu können, gegeben, wenn bestimmte physische und psychische Bedingungen erfüllt sind. Entsprechendes gilt für Zwecke, wie den, zum Essen bestimmt zu sein. Das einzige, was allen Eigenschaften gemeinsam zu sein scheint, ist die Tatsache, dass aufgrund von Eigenschaften Ähnlichkeiten zwischen Entitäten festgestellt werden, was wiederum dazu führt, dass Kategorien, also Klassen von Entitäten, gebildet werden (cf. Lakoff 1987: 5ff., 68ff.).

Wenn man sich diesen Zusammenhang bewusst gemacht hat, muss man einsehen, dass Eigenschaften an einem einzigen Objekt überhaupt nicht beobachtet werden können, sondern dass zur Beobachtung einer Eigenschaft immer (mindestens) ein Vergleichsobjekt erforderlich ist, das dem zu beschreibenden Objekt ähnlich oder unähnlich ist (cf. Ehlich 1987: 289f.). Das Ähnliche oder Unähnliche zwischen beiden, also das, aufgrund dessen sie gleichen oder entgegengesetzten Kategorien zugerechnet werden können, ist die Eigenschaft. Eigenschaften sind demnach, ebenso wie Orte, ungesättigte Relationen. Sie bestehen aus einer Beziehung der Ähnlichkeit oder Unähnlichkeit (Nähe oder Ferne) sowie einem Vergleichsobjekt und besitzen eine Leerstelle, die durch ein zu beschreibendes Objekt zu besetzen ist, ebenso wie die Leerstelle eines Ortes durch ein zu lokalisierendes Objekt besetzt werden muss. Während aber Orte ungesättigte Kontiguitätsrelationen sind, sind Eigenschaften ungesättigte Similaritätsrelationen. ${ }^{6}$

In der Sprache werden Eigenschaften durch Prädikatsausdrücke bezeichnet. ${ }^{7}$ Alle Wörter, die dazu dienen, Entitäten zu beschreiben, also Substantive, die

${ }^{6}$ Diese Definition der Eigenschaft steht im Einklang mit der Literatur über Vergleiche (cf. Hahnemann 1999: 1-8; Thurmair 2001: 108-164; auch: Blühdorn 1993: 241ff.).

${ }^{7}$ Die Prädikatenlogik notiert Eigenschaften als einstellige Prädikate, etwa die Eigenschaft blau als das Prädikat B(x). Die Variable x steht für das zu beschreibende Objekt, dem die betreffende Eigenschaft zugesprochen wird. Das Vergleichsobjekt dagegen ist 
meisten Adjektive, viele Verben usw. bezeichnen Eigenschaften, während Situierungs-, Bedingungs- und Verursachungsrelationen gerade nicht durch solche Sprachmittel, sondern durch sogenannte funktionale Kategorien wie Präposition, Konjunktion, Modalpartikel, Determinans, Tempus, Modus etc. kodiert werden. Weil aber similaritäts- und kontiguitätsrelationale Sprachmittel im wesentlichen disjunkte Mengen bilden, besitzt das Wörtchen so allerhöchsten Wert für den Linguisten, denn obwohl seine Bedeutung similaritäts- und nicht kontiguitätsrelational ist, gehört es einer funktionalen und nicht einer lexikalischen Kategorie an.

Die abstrakte relationale Bedeutung von so ist also exakt die gleiche wie die von $d a(\mathrm{O}-\mathrm{S}, \mathrm{R}, \mathrm{E})$ :

(18.a) Das neue Auto soll so aussehen.

(18.b) Das neue Regal soll da stehen.

So bedeutet "mit einer (salienten) Eigenschaft eines identifizierbaren Bezugsobjektes", $d a$ bedeutet "in der Nähe eines identifizierbaren Bezugsobjektes". In beiden Fällen wird bei realdeiktischem Gebrauch das Bezugsobjekt durch eine äußerungsbegleitende Geste gezeigt (cf. Ehlich 1987: 289; Thurmair 2001: 29f.). Es ist in der Äußerungssituation auffindbar und fungiert als Diskursanker (cf. Sandig 1987: 331), aber es ist nicht der Sprecher.

Auch in dem Satz:

(19) So lag der Tote.

zeigt so an, dass das zu beschreibende Objekt E (wahlweise der Tote oder das Liegen des Toten) zum relevanten Zeitpunkt eine Eigenschaft hatte, die einer Eigenschaft des Bezugsobjektes $\mathrm{R}$ ähnlich oder gleich ist. Nun ist es durchaus vorstellbar, dass der Sprecher, um das so zu illustrieren, sich selbst in der betreffenden Haltung an die gemeinte Stelle legt und die fragliche Eigenschaft mit dem eigenen Körper zeigt. Wie ist diese Möglichkeit mit der Beschränkung vereinbar, dass der Diskursanker nicht der Sprecher sein darf? Hier ist es nützlich, sich an Bertolt Brechts Theorie vom epischen Theater zu erinnern (cf. Brecht 1967), die auf eben solche Vorspielszenen Bezug nimmt und ihren distanzierenden, Kritik anregenden Charakter hervorhebt. Der Sprecher legt sich nicht als Sprecher an die Stelle des Toten, er tut nicht so, als ob er selbst tot wäre, sondern im Gegenteil, er muss ganz lebendig sein, um zeigen und sagen zu können, wie der Tote da lag. Er nimmt zum Zwecke des Vorspiels Abstand von sich selbst, legt seinen Körper als Bezugsobjekt auf den Boden und spricht dazu aus der Perspektive eines zuschauenden Beobachters. Brechts theaterpsychologische Ana-

konzeptueller Bestandteil von B. Es ist irgendein anderer Gegenstand, an dem die Eigenschaft beobachtet wurde und der nun gleichsam als Muster oder Prototyp für diese Eigenschaft verwendet wird. 
lyse hat ihr exaktes linguistisches Korrelat in der Semantik von so. Der zu beschreibende Referent und das Bezugsobjekt, dem er ähnelt, befinden sich, ebenso wie der Sprecher, in der Äußerungssituation, aber der Sprecher hält sich von ihnen entfernt und kann dadurch kritisch kommentieren. In diesem Punkt unterscheidet sich so erkennbar von hier, das Nähe zwischen allen vier Gliedern der Relation anzeigt (O,S,R,E). Hier wirkt identifikationsfördernd, so und $d a$ dagegen distanzierend (cf. Blühdorn 2002). ${ }^{8}$

Die semantische Struktur von Orten als ungesättigte Kontiguitätsrelationen habe ich oben anhand der Gegenüberstellung von intransitivem $d a$ und transitivem auf in auf dem Tisch herausgearbeitet. Eine entsprechende Gegenüberstellung für Similaritätsrelationen (cf. Thurmair 2001: 27f.) ist:

(20) Der Tote lag so.

(21) Der Tote lag wie ein Fragezeichen.

Wie hat zahllosen Grammatikschreibern Kopfzerbrechen bereitet, weil es einerseits einer Präposition ähnelt, andererseits keine Kasusrektion hat und sich auch semantisch anders verhält als die prototypischen Präpositionen (cf. Hentschel/Weydt 1990: 254f.; Zifonun et al. 1997: 61f.; Zifonun 1998; Di Meola 2000: 45ff.; Thurmair 2001: 41ff.). Es eröffnet eine zweistellige Similaritätsrelation, so wie auf eine zweistellige Kontiguitätsrelation eröffnet. Bei wie muss das Bezugsobjekt, mit dem der Referent eine Eigenschaft gemeinsam hat, als Komplement genannt werden, während es bei so nicht genannt werden kann. So ist intransitiv, wie transitiv. Während aber die Kontiguitätspräpositionen in, an, auf etc. ein relativ umfangreiches Inventar bilden, scheint die Similaritätspräposition wie einem viel kleineren Inventar anzugehören.

So kann, wie $d a$, nicht nur realdeiktisch, sondern auch anaphorisch verwendet werden. Ein zuvor eingeführter Referent kann mit so als Bezugsobjekt für die Beschreibung eines anderen Referenten wiederaufgenommen werden:

(22) Waldi hat vier Beine und hinten einen Schwanz. So ( $\rightarrow$ wie Waldi) sieht auch unsere Katze aus.

Bezugsobjekt ist hier ein Individuum namens Waldi, von dem wir annehmen wollen, dass es ein Hund ist, und es wird festgestellt, dass eine bestimmte Katze

\footnotetext{
${ }^{8}$ Ehlich (1987: 296ff.) und Burkhardt (1987: 303f.) beschreiben einen Gebrauch von so, bei dem durch "Deixis ins Leere" (Ehlich 1987: 292) der Leser zum "Komplizen des Autors" (ebd. 298) gemacht und damit zur Identifikation eingeladen wird. Solche Identifikationsangebote erklären sich aus der Nahrelation S,R,E und sind daher mit der von mir beschriebenen, auf $\mathrm{O}-\mathrm{S}$ beruhenden, Distanzierung vom Sprecher durchaus verträglich. Wir erkennen hier die außerordentliche Flexibilität deiktischer Sprachmittel bei der Referenzkoordination im Diskurs.
} 
ihm hinsichtlich der genannten Eigenschaft, vier Beine und hinten einen Schwanz zu haben, ähnelt.

Ferner kann so, ebenfalls wie $d a$, kataphorisch gebraucht werden (cf. Thurmair 2001: 28):

(23) Unsere Katze sieht so aus wie Waldi.

Bei nicht-realdeiktischem Gebrauch ist $s o$ in einem solchen Satz erst interpretierbar, wenn mit der wie-Phrase ein Bezugsobjekt für die Ermittlung der relevanten Eigenschaft nachgeliefert wurde.

Räumliche und zeitliche, alethische und deontische Situierungen, Bedingungsund Ursachenkonstellationen kann similaritätsrelationales so von Haus aus nicht anzeigen, da es sich hierbei um Kontiguitätsrelationen handelt. Allerdings wurde bereits festgestellt, dass Bedingungen und Zwecke auch Eigenschaften von Entitäten konstituieren können, und dasselbe gilt im Prinzip für zeitliche und räumliche Situierungen. Similarität und Kontiguität sind nicht unabänderlich wesensverschieden, sondern repräsentieren ihrerseits zwei unterschiedliche Betrachtungsweisen, die in der menschlichen Kognition angelegt sind. Dieselben Fakten in der Außenwelt können unter dem Gesichtspunkt der Ähnlichkeit (symmetrisch) oder unter dem der Angrenzung (nicht-symmetrisch) betrachtet werden.

Die deutsche Sprache gibt diese Beobachtung präzise wieder, indem sie uns erlaubt, alternativ zu sagen (cf. Redder 1987):

(24.a) Wenn wir uns nicht beeilen, dann kommen wir zu spät.

(24.b) Wenn wir uns nicht beeilen, so kommen wir zu spät.

Die deutschen $d$-Deiktika wie $d a$, dies-, dann etc. zeigen (nicht-symmetrische) Kontiguitätsrelationen an. Dann in (24.a) kann paraphrasiert werden durch "unter der genannten Bedingung". Die $s$-Deiktika wie so und solch ${ }^{-}{ }^{9}$ dagegen zeigen (symmetrische) Similaritätsrelationen an. So in (24.b) ist zu paraphrasieren durch "in einer Situation, die so beschaffen ist; unter einer solchen Bedingung". Aus beiden Feststellungen dürften sich in den meisten Kontexten die gleichen Schlussfolgerungen ergeben, so dass Paare wie (24.a/b) leicht synonym erscheinen. Das geht so weit, dass auch bei so, ebenso wie bei dann, Situierungs-, Bedingungs- und Verursachungs-Lesarten verfügbar werden. Der semantische Unterschied zwischen kontiguitätsrelationalem dann und similaritätsrelationalem so wird durch die Pragmatik, d.h. durch kontextgebundene Inferenzen, verwischt.

\footnotetext{
${ }^{9}$ Nicht zu verwechseln mit einer anderen, ebenfalls als $s$-Deiktika bezeichneten Gruppe mit kontiguitätsrelationaler Semantik, zu der sie, sich, sein etc. gehören.
} 
De facto ist der semantische Unterschied zwischen dann und so übrigens sogar noch größer, als bis jetzt angedeutet. Dann hat nämlich nicht die gleiche relationale Bedeutung wie $d a$ und so, sondern zeigt wie dort Distanz zwischen dem Sprecher als Diskursanker und dem Bezugsobjekt an, in dessen Nähe der aktuelle Referent situiert wird. Seine Relationsformel ist O,S-R,E. Dies führt zu der Opposition zwischen $d a$ und dann in Paaren wie:

(25.a) Als wir uns nicht beeilten, da kamen wir zu spät.

(25.b) Wenn wir uns nicht beeilten, dann kamen wir zu spät.

Es ist vereinzelt beobachtet worden, dass so in Sätzen wie (24.b) eher darauf hinweist, dass die mit wenn eingeführte Bedingung erfüllt ist, während dann in Sätzen wie (24.a) dies offen lässt (cf. Redder 1987: 321ff.). Der Eindruck der offenen Bedingung bei dann geht semantisch auf die Distanz des Bezugsobjektes zum Sprecher als Diskursanker zurück $(\mathrm{O}, \mathrm{S}-\mathrm{R})$, während der Eindruck der erfüllten Bedingung bei so sich durch die Nähe zwischen Bezugsobjekt und Diskursanker erklärt, auch wenn letzterer nicht der Sprecher ist (O-S,R). Dass $d a$ in Sätzen wie (25.a) die Interpretation der erfüllten Bedingung nicht nur nahelegt, sondern erzwingt, und wie (25.b) sich von (24.a) unterscheidet, werden wir besser verstehen, wenn wir die Bedeutung von als und dessen Opposition zu wenn studiert haben.

In Bezug auf so können wir festhalten, dass es in Paaren wie (24.a/b) infolge pragmatischer Gleichwertigkeit kontiguitätsrelational interpretiert werden kann. Per Analogie mag es allmählich die Fähigkeit angenommen haben, auch in anderen Kontexten Kontiguitätsrelationen anzuzeigen. So ist zu erklären, dass $s o$, genau wie $d a$, transitiviert werden kann, indem es den Ausdruck, der das Bezugsobjekt bezeichnet, als Komplement nimmt:

(26) Wenn man den Medien glauben darf, so leben wir immer mehr in einer Spaßgesellschaft.

(27) So man den Medien glauben darf, leben wir immer mehr in einer Spaßgesellschaft. ${ }^{10}$

In seiner transitiven Variante als subordinierende Konjunktion ist so kaum noch similaritätsrelational zu interpretieren. Die bevorzugte Lesart ist hier eindeutig kontiguitätsrelational. Das bedeutet, dass die ursprüngliche semantische Opposition zu $d a$ neutralisiert ist. Dennoch sind transitives so und transitives $d a$ keineswegs synonym, d.h. hier muss eine neue semantische Opposition aufgekommen sein. Vergleichen wir dazu Beispiel (28):

${ }^{10}$ Dass eine entsprechende Transitivierung mit dann nicht möglich ist, ist ein weiterer Beleg dafür, dass der semantische Unterschied zwischen dann und so größer ist, als Paare wie $(24 . \mathrm{a} / \mathrm{b})$ suggerieren. 
(28) Da man den Medien glauben darf, leben wir immer mehr in einer Spaßgesellschaft.

Offenbar stehen transitives $s o$ und $d a$ in einer Definitheitsopposition. $D a$ zeigt an, dass das Bezugsobjekt (hier: die Bedingung) im Kontext verifiziert werden kann (hier: als erfüllt bekannt ist). Im Gegensatz dazu steht transitives so der Indefinitheit von wenn näher. Allerdings scheint es bezüglich der Verifiziertheit der Bedingung nicht ebenso offen wie wenn zu sein, sondern zumindest die Tendenz anzuzeigen, an ihre Erfülltheit zu glauben. Außerdem zeigt so (wie $d a$ ) an, dass die Bedingung für die Folge hinreichend ist, was bei wenn nicht gelten muss. Wenn markiert lediglich den Bezugssachverhalt als Bedingung und lässt die semantische Beziehung zum Bedingten offen (cf. Diessel 1996). Kontiguitätsrelationales wenn, so und $d a$ bilden demnach eine Skala, auf der $d a$ volle Definitheit, wenn volle Indefinitheit und so eine Art schwacher Definitheit anzeigen. Dieser Sachverhalt kann durch Relationsformeln abgebildet werden:

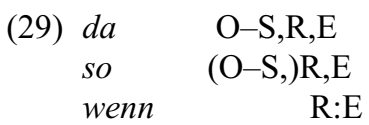

Definitheit bedeutet nichts anderes als die Rückbindung von $\mathrm{R}$ an den Äußerungskontext und den Sprecher. Bei $d a$ ist eine solche Rückbindung in vollem Umfang gegeben, bei wenn ist sie gar nicht gegeben, bei transitivem so ist sie abgeschwächt. Bei wenn ist darüber hinaus die Relation zwischen $\mathrm{R}$ und $\mathrm{E}$ nicht auf Nähe oder Ferne festgelegt. Nicht-Festgelegtheit einer Relation zeige ich durch einen Doppelpunkt an.

Wenn wir transitives so mit intransitivem vergleichen, so erkennen wir, dass der Transitivierungsvorgang hier, anders als bei $d a$, mit einer semantischen Reduktion (Abschwächung der Definitheit) einhergeht.

\section{Beschreibendes und schlussfolgerndes also}

Als stammt nicht direkt, sondern über die Zwischenstufe al(l)so von so ab (cf. Kluge 1975: 16; Paul 1992: 24f.). All- im Sinne von "ganz" hat hier offenbar Verstärkungsfunktion wie in allhier und allda (cf. Anderson et al. 1989: 854). Also hat sich neben so und als als eigene Form mit eigener Bedeutung bis in die Gegenwartssprache hinein erhalten.

Für realdeiktische Verwendung von $a l(l)$ so habe ich keine eindeutigen Belege. Die Darstellung in Anderson et al. (1989: 853ff.) lässt es aber sicher erscheinen, dass sie in frühneuhochdeutscher Zeit möglich war. Seine Bedeutung dürfte weitgehend der von intransitivem so entsprochen haben (cf. auch Paul 1992: 25).

Anaphorisches und kataphorisches allso finden sich in den folgenden Belegen: 
(30) (...) Weil wir in Pilgramschaft des Lebens müssen bleiben /

So reicht die Hoffnung uns das beste ReiseBrodt.

Wir liessen uns allso den Schluß des Himmels leiten /

Der uns geführet hat durch Berge See / und Land / (...)

(Christian Hofmann von Hofmannswaldau, TrauerGedicht bey Absterben eines vertrauten Freundes)

(31) Ich halte es aber alles vor bueben stükh und Herzog Ernst vor so schlimm alls seine herrn principales; wann er mit mir allso geredet hett wie mit E.L. Ich maine, Ich weere ihm greilih uber das maul gefahren (...)

(Kaiser Ferdinand III. an seinen Bruder Erzherzog Leopold Wilhelm, 1640; http://home.pages.at/ek-ledel/diplom/202.htm\#155 - 30.08.2002)

Der kataphorische Gebrauch in Beispiel (31) entspricht der weiter oben für kataphorisches so gegebenen Analyse (cf. Beispiel (23)). Der anaphorische Gebrauch in Beispiel (30) ähnelt eher dem an wenn anschließenden Gebrauch von so in (24.b). Allso nimmt einen zuvor erwähnten Referenten (uns) als Bezugsobjekt wieder auf, interpretiert den Sachverhalt, an dem er teilnimmt (reicht die Hoffnung uns das beste ReiseBrodt), als seine Eigenschaft und verwendet diese zur Beschreibung eines zweiten, weiter gehenden Sachverhaltes, an dem der gleiche Referent teilnimmt (Wir liessen uns den Schlu $\beta$ des Himmels leiten). Neben dieser charakterisierenden, similaritätsrelationalen Lesart ist jedoch auch eine bewertende Deutung möglich, derzufolge der Nachsatz Wir liessen uns allso den Schluß des Himmels leiten die in den Vorgängersätzen beschriebene Sachlage schlussfolgernd beurteilt. Die Schlussfolgerungsrelation ist aber eine Kontiguitätsrelation, d.h. auch bei also können wir den gleichen Übergang nachvollziehen, der oben für so aufgewiesen wurde (auch das an weil anschließende kausale $s o$ in (30) ist kontiguitätsrelational). Die Ambiguität des Beispiels illustriert den semantischen Zusammenhang zwischen beschreibendem so und schlussfolgerndem also.

Die typische Verwendungsweise von also in der Gegenwartssprache wird durch die Formel $p$ (und $q$ ), also $r$ wiedergegeben:

(32) Ich esse, also bin ich.

$\mathrm{Zu}$ situierende Objekte und Bezugsobjekte der durch also angezeigten Schlussfolgerungs-Relationen sind Propositionen oder Akte. Die Folgerungen betreffen deren Wahrheits- bzw. Erwünschtheitswerte. Raum- oder Zeitrelationen können mit also nicht kodiert werden. Also bedeutet so viel wie "unter solchen/diesen Bedingungen", wobei der Unterschied zwischen Similarität und Kontiguität, wie bei transitivem so neutralisiert ist bzw. Similarität in Kontiguität umgedeutet wird.

Auch für also gilt die Relationsformel O-S,R,E. Das zu situierende Objekt E (eine Proposition oder ein Akt) wird hinsichtlich seines Wahrheits- bzw. Er- 
wünschtheitswertes vom Bezugsobjekt (einer anderen Proposition / einem anderen Akt oder einer Menge von Propositionen bzw. Akten) festgelegt. Das Bezugsobjekt bzw. -system ist im Kontext gegeben (im allgemeinen durch Vorerwähntheit), ist aber vom Sprecher unabhängig.

Anders als so kann also gegenwartssprachlich keine Nebensätze einbetten. Im Frühneuhochdeutschen war aber auch transitive Verwendung von also möglich (cf. Anderson et al. 1989: 855). An dessen Stelle sind gegenwartssprachlich transitives als, so und wenn getreten. Also kann sich gegenwartssprachlich aber aus der syntaktischen Struktur des Satzes lösen und zur selbstständigen Diskurspartikel werden:

(33) Ich bin seit dem 18.09.01 hier angemeldet und habe bis jetzt 2 oder 3 Mails bekommen, also ich habe damit kein Problem.

(http://www.teltarif.net/forum/x-feedback/766-8.html - 05.09. 2002)

In dieser Verwendungsweise bleibt zwar ein (vage) schlussfolgernder Charakter erhalten, aber die Beziehung zwischen Prämisse(n) und Folgerungen wird unverbindlicher. Die Prämissen müssen nämlich für die Folgerung nicht mehr hinreichend sein. Oft dient also als Diskurspartikel zur bloßen Markierung eines strukturierenden Neuansatzes, etwa im Sinne von "in diesem Zusammenhang sage ich folgendes" (cf. dazu auch Paul 1992: 26):

(34) Ich sitze hier grade verzweifelt an dem Versuch meinen Strafrechtsschein zu packen und brauche dringend Hilfe! (...) Folgender Fall. (...) Wie haben sich $\mathrm{C}$ und V strafbar gemacht? Also ich habe mir folgendes überlegt. (...)

(http://www.jurawelt.com/pipermail/studenten/20011016/001157.html - 05.09.2002)

Während bei so im Grammatikalisierungs-Prozess die deiktische Rückbindung abgeschwächt wird, neutralisiert sich bei also die Relation zwischen $\mathrm{R}$ und $\mathrm{E} \mathrm{zu}$ O-S,R:E. Die deiktische Rückbindung bleibt dagegen erhalten, wie die Beispiele (33) und (34) klar zeigen.

\section{Temporales als}

Wir sind nun endlich darauf vorbereitet, uns der Analyse von als zuzuwenden. Aufgrund der sprachhistorischen Gegebenheiten liegt es nahe, dessen Semantik vor dem Hintergrund von so und also zu rekonstruieren. Formale Erosion kennzeichnet als gegenüber also als Produkt fortgeschrittener Grammatikalisierung. Ebenso wenig wie so durch also ist aber also durch als sprachhistorisch ersetzt worden, sondern die älteren Elemente sind jeweils neben den jüngeren erhalten geblieben. Im Inventar der similaritätsrelationalen Konnektoren hat mithin eine zweistufige Erweiterung stattgefunden: 
(35)
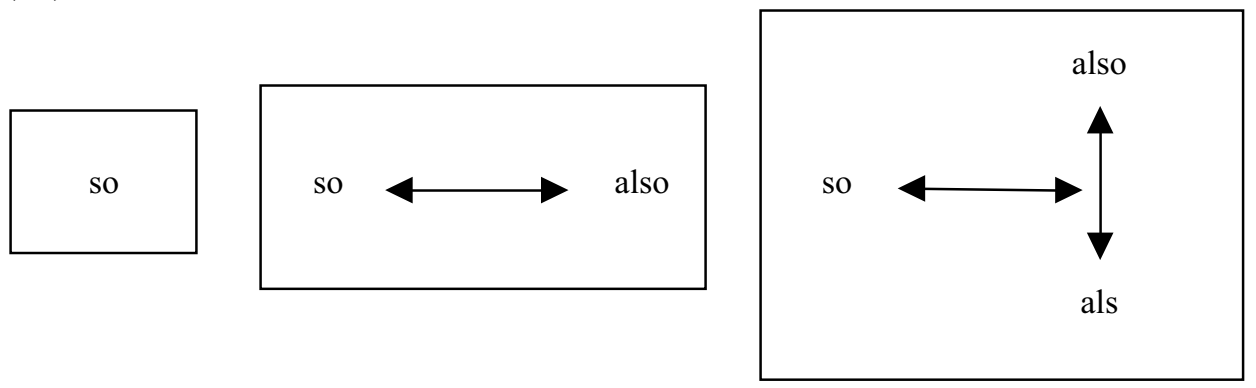

Auch temporales als kann, wie so und also, transitiv und intransitiv verwendet werden. Intransitiver Gebrauch kommt vor allem im südwestdeutschen Sprachraum vor (cf. auch Rünneburger 1998):

(36) Aci: Aber am Samstag in Holzhausen konntest du wieder saufen oder was? Flo: Ja. ... Moment mal. Das wagst DU MICH zu fragen? Wer ist denn die ganze Zeit darum gefallen und hat als gesagt: "Ich muss gleich kotzen."? (http://www.abi02.de/lts/sprueche/12.html - 22.09.2002)

Dieses als, das näherungsweise durch "immerfort, unausgesetzt" ("die ganze Zeit") paraphrasiert werden kann, wird von Kluge (1975: 16) als separates Lemma geführt und etymologisch aus dem Akkusativ Singular Neutrum von all(es) hergeleitet. Es sei "nur lautlich (...) mit der Konjunktion als zusammengefallen" (ebd.; cf. auch Paul 1992: 25). Demnach wäre als in Sätzen wie (36) ein temporaler Quantor, dessen Bedeutung nicht in Analogie zu so und also erfasst werden kann.

Es gibt aber auch eine (archaische) Variante von intransitivem als, die sehr wohl an so und also anschließbar ist (cf. Paul 1992: 24):

(37) Geh ich in mein Kämmerlein, Will mein Bettlein machen; Steht ein bucklicht Männlein da, Fängt als an zu lachen. (Des Knaben Wunderhorn)

In diesem Beispiel werden zwei Sätze semantisch miteinander verknüpft. Der Vordersatz ist ein uneingeleiteter Nebensatz, der temporal und konditional interpretiert werden kann und den Bezugssachverhalt R kodiert. Der Nachsatz mit intransitivem als kodiert den situierten Sachverhalt E, der durch als offenbar in temporaler bzw. konditionaler Nähe zu R situiert wird. R ist zugleich der durch als anaphorisch wiederaufgenommene, durch Vorerwähntheit im Kontext identifizierbare, aber vom Sprecher entfernte Diskursanker. Die Bedeutung dieser Variante von intransitivem als kann somit präzise durch die Relationsformel $\mathrm{O}-\mathrm{S}, \mathrm{R}, \mathrm{E}$ wiedergegeben werden. 
Als in (37) ist durch alsbald paraphrasierbar. Man denke dazu auch an andere bedeutungsverwandte Adverbien wie alsda und alsdann (cf. Anderson et al. 1989: 849ff.). Wahrscheinlich handelt es sich bei der intransitiven als-Variante in (37) um eine Verkürzung aus einem solchen zusammengesetzten Adverb. Denn etymologisch scheint als, anders als $d a$, von Haus aus transitiv zu sein (cf. ebd. 842ff.). Allerdings kann Beispiel (37), ebenso wie es weiter oben für $d a$ und so demonstriert wurde, unter weitgehender Erhaltung seiner Bedeutung (wenn auch unter Einbuße an poetischer Qualität) umgestellt werden zu:

(37.a) Als ich in mein Kämmerlein geh und mein Bettlein machen will, steht ein bucklicht Männlein da und fängt an zu lachen.

In dieser Fassung nimmt als den Teilsatz, der den Bezugssachverhalt R kodiert, als Komplement. $\mathrm{Ob}$ die relationale Bedeutung der Konjunktion als ebenfalls durch die Formel O-S,R,E wiederzugeben ist oder ob, ähnlich wie bei transitivem so, eine semantische Reduktion stattgefunden hat, soll im folgenden untersucht werden.

\subsection{Paradigmatische Oppositionen}

Der zeitrelationale Gebrauch der Konjunktion als wird durch folgendes Beispiel illustriert:

(38) Als ich hereinkam, spielte sie Klavier.

Gehen wir zunächst von der Hypothese aus, dass die Bedeutung von als hier ebenfalls der Formel O-S,R,E entspricht. Thurmair (2001: 67 u.ö.) schreibt als in allen seinen Verwendungsweisen die semantische Grundfunktion "Identifizierung" zu, die zwar weniger spezifisch ist, aber mit dieser Formel gut zusammenstimmt. Das zu situierende Objekt E ist der im Hauptsatz dargestellte Sachverhalt (hier: sie spielte Klavier). Das Komplement von als (der temporale Nebensatz) gibt den Bezugssachverhalt $\mathrm{R}$ an (hier: ich kam herein). Dieser ist zugleich der Diskursanker S. Der Diskursanker darf nicht das Sprechereignis sein. Man kann also nicht sagen:

(39) Als ich jetzt gerade spreche, kommt Maria herein.

Die semantischen Unterschiede zwischen als und anderen Konnektoren ergeben sich aus Gebrauchsbedingungen, die man in den Grammatiken etwa folgendermaßen dargestellt findet (cf. Hentschel/Weydt 1990: 275):

1. Als zeigt Relationen der zeitlichen Situierung, also Relationen zwischen Sachverhalten an (cf. Heidolph et al. 1984: 403). Hinsichtlich dieser Eigenschaft steht es in Opposition zu transitivem $d a$ (nur archaisch temporal situierend, heute bevorzugt konditional verwendet) und so (nur konditional verwendbar). 
2. Als zeigt Gleichzeitigkeit des situierten Ereignisses und des Bezugsereignisses an. In dieser Hinsicht kontrastiert es mit bevor (Vorzeitigkeit) und nachdem (Nachzeitigkeit) (cf. Heidolph et al. 1984: 790; Duden 1998: 405).

3. Als zeigt Vorzeitigkeit des Bezugsereignisses gegenüber dem Sprechereignis an. Dies zeigt sich in der bevorzugten Kombination mit Vergangenheitstempora. In dieser Hinsicht kontrastiert es mit wie (Nicht-Nachzeitigkeit) und mit wenn (neutral) (cf. Zifonun et al. 1997: 1147; Duden 1998: 798f.).

4. Als zeigt punktuelle oder einmalige Bezugsereignisse an. In dieser Hinsicht kontrastiert es mit wenn (neutral) (cf. Zifonun et al. 1997: 2284f.; Duden 1998: 799).

Punkt 1., der die Oppositionen zu da und so betrifft, ist unstrittig. Punkt 2. wird bei Zifonun et al. (1997: 1145) dahingehend präzisiert, dass als nicht eigentlich Gleichzeitigkeit, sondern zeitliche Überlappung des situierten Ereignisses mit dem Bezugsereignis anzeigt. Beide Möglichkeiten werden durch die Teilformel $R, E$ (für eine Nah-Relation) erfasst.

Zu Punkt 3. erläutern Zifonun et al. (1997: 1147f., 2285), dass als in Fällen wie (40.a) eine Vergangenheits-Interpretation des Präsens erzwingt:

(40.a) Als Cäsar den Rubikon überschreitet, ist das Ende der römischen Republik nahe.

Ich glaube nicht, dass diese These richtig ist. Über das erwähnte Bezugsereignis, das Überschreiten des Rubikon durch Cäsar, enthält unser allgemeines Weltwissen die Information, dass es vor dem Sprechereignis stattgefunden hat. Zum damaligen Zeitpunkt gab es noch nicht einmal die Sprache, in der (40.a) formuliert ist. Wir müssten das Präsens in diesem Satz also auch dann mit Vergangenheits-Interpretation versehen, wenn die Konjunktion als nicht vorkäme:

(40.b) Cäsar überschreitet den Rubikon. In diesem Augenblick ist das Ende der römischen Republik nahe.

Niemand würde vernünftigerweise behaupten wollen, das Demonstrativum diesem erzwinge in (40.b) eine Vergangenheits-Lesart des Präsens.

Die Semantik von als kann besser an Beispielen studiert werden, die nicht durch Weltwissen belastet sind:

(41.a) Als er viel Geld verdiente, war er schlecht gelaunt.

(41.b) Als er viel Geld verdient, ist er schlecht gelaunt.

(41.b) erlaubt eine Vergangenheits-Interpretation, erzwingt sie aber nicht. Erzwungen wird lediglich, dass das Bezugsereignis (er verdient viel Geld) vom Sprechereignis entfernt ist, was ich durch die Teilformel O-S,R ausgedrückt habe. Diese Entfernung kann auch textuell durch die Kohärenz einer Erzählung 
hergestellt werden, bei der es überhaupt nicht darauf ankommt, in welcher zeitlichen Relation die besprochenen Ereignisse zum Sprechereignis stehen:

(42) Stellen wir uns einen ehrgeizigen jungen Mann namens Erwin vor. Er absolviert das Gymnasium mit einem glänzenden Abitur, studiert Betriebswirtschaft, macht in acht Semestern seinen Doktor und findet einen Traumjob in einem multinationalen Konzern. Aber als er viel Geld verdient, ist er schlecht gelaunt.

In diesem Beispiel wird durch den Textzusammenhang eine VergangenheitsInterpretation praktisch ausgeschlossen. Hier wird Entfernung zum Sprechereignis durch Fiktionalität und narrative Kohärenz erzeugt. Als verankert das Bezugsereignis (er verdient viel Geld) in einer erzählten fiktionalen Ereignissequenz (er absolviert das Gymnasium, studiert, macht seinen Doktor). Der Diskursanker S, dessen Notwendigkeit für die Bedeutungsbeschreibung an solchen Beispielen besonders deutlich wird, muss in dieser Ereignissequenz und gerade nicht in der Äußerungssituation aufgesucht werden. Wie das deutsche Präteritum unter be-stimmtem Blickwinkel als Erzähltempus charakterisiert werden kann (cf. Ehrich 1992: 100; Weinrich 1993: 219f.), so ist als unter ähnlichem Blickwinkel eine Erzählkonjunktion (cf. Schanen/Confais 1989: 498).

$\mathrm{Zu}$ Punkt 4. muss zunächst nachgefragt werden, was genau unter Punktualität und Einmaligkeit zu verstehen ist. Betrachten wir dazu folgende Beispiele:

(43) Auch als die Atombombe noch nicht erfunden war, kannten die Menschen die Existenzangst. (nach Heidolph et al. 1984: 406)

(44) Es war wie jeden Tag: Als Elke zu Bett ging, saß Dennis noch am Computer.

Sicherlich ist der Zustand, in dem die Atombombe noch nicht erfunden war, unwiederholbar und daher in gewissem Sinne einmalig, aber er hat Hunderttausende von Jahren angedauert, kann also auf keinen Fall als punktuell gelten. Umgekehrt mag das Ereignis des Zu-Bett-Gehens in Beispiel (44) punktuell sein, aber Einmaligkeit wird explizit ausgeschlossen.

Die an sich richtigen Intuitionen der Grammatikschreiber müssen also anders formuliert werden: Als zeigt an, dass ein spezifischer Bezugssachverhalt gewählt wird, dessen Faktizität vorausgesetzt ist. Man erkennt dies sehr deutlich in der Gegenüberstellung zu wenn, das gerade diese Informationen nicht anzeigt:

(45) Wenn er viel Geld verdient, ist er schlecht gelaunt.

(46) Wenn er viel Geld verdiente, war er schlecht gelaunt.

(47) Wenn er viel Geld verdiente, wäre er schlecht gelaunt.

In (45) bis (47) werden unspezifische Bezugssachverhalte gewählt. Die Frage ihrer Faktizität bleibt offen. Im Präsens-Satz (45) sind unterschiedliche Interpretationen möglich, z. B. eine konditionale Deutung im Sinne von falls, eine temporale Deutung mit Zukunftsbezug, entweder auf eine einzige oder auf mehrere zukünftige Situationen bezogen, eine distributiv totalisierende Deutung im 
Sinne von jedesmal wenn, eine kumulativ totalisierende Deutung im Sinne von immer wenn oder eine generische Deutung im Sinne der Zuschreibung einer Eigenschaft an den Subjektsreferenten. In (46) wird die Verbform des wenn-Satzes durch den Obersatz als Präteritum disambiguiert. Hier ist die konditionale Interpretation im Sinne von falls mit Vergangenheitsbezug ${ }^{11}$ möglich, ferner die beiden totalisierenden und die generische Deutung, ebenfalls mit Vergangenheitsbezug, nicht aber die temporale Deutung mit Zukunftsbezug. In (47) wird die Verbform als Konjunktiv II disambiguiert. Dadurch erhält der Satz hypothetischen Charakter: Faktizitäts-Neutralität wird durch Nicht-Faktizität ersetzt. Möglich bleibt hier vor allem eine konditionale Deutung im Sinne von falls. Temporale Deutungen mit Zukunftsbezug, sowie totalisierende und generische Deutungen scheinen dagegen auszufallen.

Blicken wir von hier aus auf die Paare (24.a/b) und (25.a/b) zurück, bei deren Diskussion weiter oben noch Fragen offen geblieben waren:

(24.a) Wenn wir uns nicht beeilen, dann kommen wir zu spät.

(24.b) Wenn wir uns nicht beeilen, so kommen wir zu spät.

(25.a) Als wir uns nicht beeilten, da kamen wir zu spät.

(25.b) Wenn wir uns nicht beeilten, dann kamen wir zu spät.

Vor dem Hintergrund der gegebenen Analyse von als und wenn erkennen wir, dass in (25.a) die Interpretation der erfüllten Bedingung (bzw. des faktischen Sachverhaltes) nicht erst durch $d a$, sondern bereits durch als erzwungen wird. $D a$ nimmt diese Information bloß anaphorisch wieder auf und bestätigt sie. Ähnlich werden die Unterschiede in der Interpretation von dann in (24.a) und (25.b) durch wenn in Verbindung mit unterschiedlichen Tempora induziert. Das Präsens in (24.a) erlaubt es, die Erfülltheit der Bedingung gänzlich offen zu lassen. Durch das Präteritum in (25.b) dagegen fallen Gegenwarts- und ZukunftsLesarten aus. Für die verbleibenden Vergangenheits-Lesarten verlangt das Präteritum sequenzielle Kontextualisierung (in einer Erzählung). Unter dieser Bedingung ergeben sich Inkohärenzen, wenn die Erfülltheit der Bedingung nicht geklärt ist. Es muss nicht an eine spezifische Situation gedacht werden, in der die Bedingung erfüllt war, aber wenn nicht angenommen wird, dass sie zu irgendeinem Zeitpunkt erfüllt war, dann ist (25.b) semantisch abweichend.

Für wenn hatte ich bereits unter (29) die Relationsformel R:E gegeben. Ihr fehlt die diskurssemantische Rückbindung an den Sprecher, die durch die Teilformel $\mathrm{O}-\mathrm{S}, \mathrm{R}$ ausgedrückt wird, d.h. wenn ist im Gegensatz zu $d a$, so und als nicht deiktisch. Bei als konkretisiert sich die deiktische Komponente in der Anzeige

${ }^{11}$ Das Präteritum ist nicht auf Vergangenheits-Lesarten festgelegt, sondern kann ebenfalls als Anzeiger von Fiktionalität interpretiert werden (cf. Weinrich 1993: 219f.). Wenn ich im folgenden vereinfachend von Vergangenheitsbezug spreche, so sind solche Interpretationen stillschweigend mitgemeint. 
von Spezifizität und vorausgesetzter Faktizität des Bezugssachverhaltes. Das ist eine schwache Form der Deixis. Als zeigt nicht, wie $d a$, an, dass der Bezugssachverhalt im Kontext identifizierbar (definit) ist, sondern lediglich, dass es einen spezifischen Bezugssachverhalt gibt. Hierbei muss es sich um einen Sachverhalt handeln, der seiner Art (Kategorie) nach im Kontext erwartbar oder erschließbar ist, der also durch Similarität kontextuell rückgebunden ist. (Wir erinnern uns daran, dass Kategorisierung auf Similarität beruht und dass als von dem Similaritätsdeiktikum so abstammt.) Präzise auf diese Eigenschaft, kategoriale Erwartbarkeit des Bezugssachverhaltes zu fordern, ist es zurückzuführen, dass als Ereignissequenzen bildet, also als Erzählkonjunktion betrachtet werden kann.

Als kann keinen Bezugssachverhalt einführen, der nicht in einer nachvollziehbaren Similaritätsbeziehung zum Kontext steht. Diese Behauptung muss noch genauer erläutert werden. Für viele $a l s$-Sätze, wie (40.a), (43) und andere bereits diskutierte Beispiele, kann eine Similaritäts-Rückbindung aufgrund allgemein verfügbaren Weltwissens mühelos und unbemerkt ad hoc konstruiert werden. Deshalb fällt die Beschränkung auf kategorial erwartbare Bezugssachverhalte gewöhnlich gar nicht auf. Denken wir aber an Fälle wie den berühmten Anfang des Grimmschen Märchens Der Froschkönig (in einer häufig zitierten, wiewohl nicht ganz originalgetreuen Fassung):

(48) In den alten Zeiten, als das Wünschen noch geholfen hat, lebte ein König, dessen Töchter waren alle schön.

Nehmen wir an, der Textanfang lautete einfach:

(49) Als das Wünschen noch geholfen hat, lebte ein König, dessen Töchter waren alle schön.

In dieser Variante hätten wir große Schwierigkeiten, dem als-Satz Sinn zu geben, und zwar präzise, weil der Sachverhalt, den er beschreibt, seiner Art nach ohne weitere Hilfestellung nicht erwartbar und somit nicht aufgrund von Similarität im Kontext verankerbar ist (jedermann weiß ja, dass das Wünschen normalerweise nicht hilft). Eben deshalb stellt der Märchenerzähler die zusätzliche realdeiktische Zeitbestimmung in den alten Zeiten voran, durch die der im alsSatz beschriebene unerwartete Sachverhalt in einem Kontext situiert werden kann, in dem möglicherweise andere als die uns vertrauten Regeln gelten und der es deshalb erlaubt, ad hoc eine Similaritäts-Rückbindung zu erzeugen. (49) ist also präzise wegen fehlender Similaritäts-Rückbindbarkeit semantisch abweichend, und (48) ist entsprechend wegen vorhandener Rückbindbarkeit semantisch wohlgeformt.

Welche semantischen Oppositionen unterscheiden nun als von da, so und wenn? Während $d a$ Identifizierbarkeit des Bezugssachverhaltes durch Kontiguitätsbeziehungen anzeigt, fordert als Spezifizität und kategoriale Erwartbarkeit des 
Bezugssachverhaltes durch Similaritätsbeziehungen. Da als aber gleichzeitig den besprochenen Sachverhalt E relativ zum Bezugssachverhalt temporal situiert, zeigt es uns dieselbe schillernde Unbestimmtheit zwischen Similarität und Kontiguität, die wir bereits bei transitivem so und bei also beobachtet haben. Entsprechend können wir die unter (29) für $d a$, so und wenn beschriebene Skala durch als erweitern:

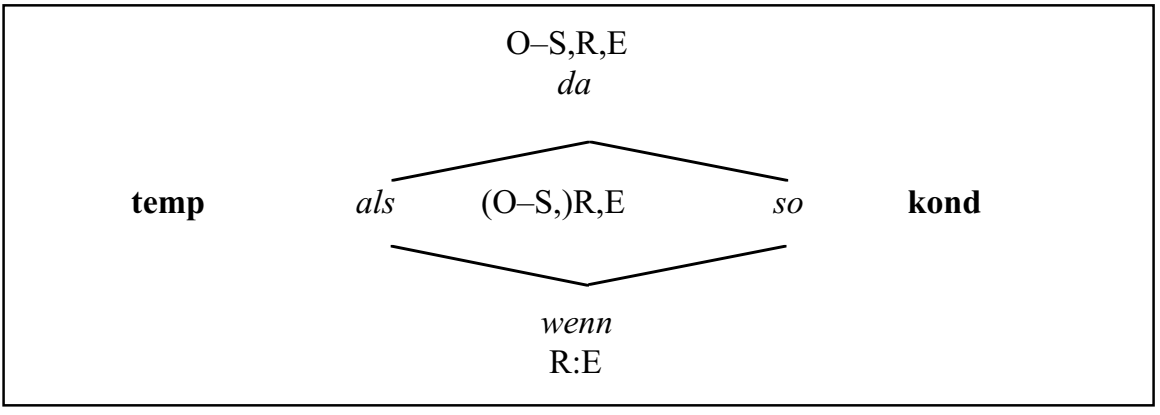

Das $s$-Deiktikum als verlangt, anders als wenn, eine kontextuelle Rückbindung, aber diese muss nicht ebenso eindeutig sein wie beim $d$-Deiktikum $d a$.

Wenn in archaisierender Sprechweise $d a$ als temporale Konjunktion anstelle von als verwendet wird, wie in Beispiel (3):

(3) Da sie ihre Nägel wieder in Ulrichs Arm eingrub, tat sie es vielleicht zu stark. (Musil; zit. nach Duden 1998: 405),

so wird anstelle eines kategorial erwartbaren ein identifizierbarer Bezugssachverhalt ausgewählt. Identifizierbare Sachverhalte können zugleich kategorial erwartbar sein, müssen es aber nicht. Das gleiche gilt umgekehrt. Identifizierbare und kategorial erwartbare Sachverhalte bilden innerhalb der Gesamtmenge der Sachverhalte $\{\mathrm{SV}\}$ sich schneidende Teilmengen $\{\mathrm{I}\}$ und $\{\mathrm{K}\}$ :

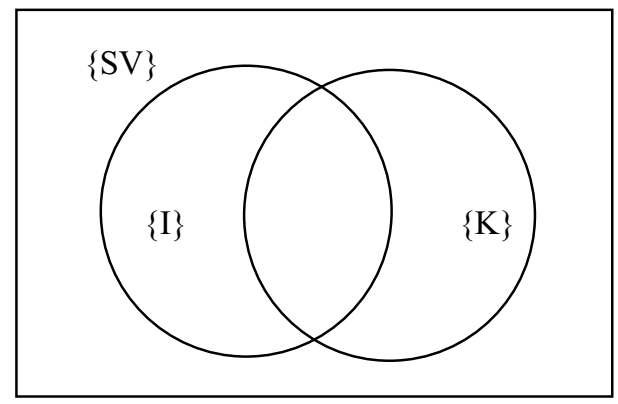


Entsprechend überschneiden sich die Anwendungsbereiche der temporalen Konjunktionen $d a$ und als, wenn man einmal von ihrer unterschiedlichen sprachgeschichtlichen Charakteristik absieht.

Würde man dagegen so anstelle von als einsetzen, so wechselte man bei gleichbleibender abstrakter Bedeutung die relationale Perspektive:

(52) So sie ihre Nägel in Ulrichs Arm eingrub, tat sie es vielleicht zu stark.

Da transitives so sprachgeschichtlich neben als erhalten geblieben ist, konnte hier eine zusätzliche semantische Opposition aufkommen. Als dient zur temporalen Situierung eines Sachverhaltes in Bezug auf einen kategorial erwartbaren Bezugssachverhalt, während transitives so nur noch konditional interpretiert werden kann, also den kategorial erwartbaren Bezugssachverhalt als Bedingung für den zu situierenden Sachverhalt kennzeichnet. Demgegenüber können $d a$ und wenn sowohl temporal-situierend als auch konditional verwendet werden. Bei $d a$ ist die temporal-situierende Lesart archaisch. Bei wenn, das ja nichtdeiktisch ist, hängt die temporale Interpretation von den gleichzeitig verwendeten Tempora ab (siehe oben, Beispiele (45) bis (47)). In (53) kommt es so zu einer konditionalen Vergangenheitsinterpretation:

(53) Wenn sie ihre Nägel in Ulrichs Arm eingrub, tat sie es vielleicht zu stark.

Bei wenn muss also die deiktische Rückbindung durch andere Sprachelemente geleistet werden.

Wir können die bisher angestellten Überlegungen zum paradigmatischen Status von als nun noch erweitern, indem wir die Opposition zwischen als und der Konjunktion wie thematisieren. Nach Duden (1998: 798f.) zeigt als Vorzeitigkeit und wie Nicht-Nachzeitigkeit des Bezugssachverhaltes gegenüber dem Sprechereignis an. Betrachten wir dazu folgende Beispiele:

(54) Als wir nach Hause kamen, war die Tür geöffnet.

(55) Als wir nach Hause kommen, ist die Tür geöffnet.

(56) Wie wir nach Hause kamen, war die Tür geöffnet.

(57) Wie wir nach Hause kommen, ist die Tür geöffnet.

(54) exemplifiziert den Vergangenheits-Gebrauch von als. Für Sätze wie (55) verlangt Duden (1998: 799) wie auch Zifonun et al. (1997: 1147) Vergangenheits-Interpretation des Präsens. Dagegen habe ich gezeigt, dass die Vorzeitigkeits-Lesart nur eine von mehreren möglichen Realisierungen der von als geforderten Distanz zwischen Diskursanker und Sprecher ist. Alternativ kann sie etwa auch durch Fiktionalität hergestellt werden. Beispiel (56) ist nach Duden (1998: 798) "umgangssprachlich". Dazu werden allerdings mehrere literarische Beispiele gegeben, die diese Einschätzung zumindest überprüfenswert erscheinen lassen. Beispiel (57) schließlich exemplifiziert nach Duden (ebd.: 799) 
einen "gewöhnlichen" Gebrauch von wie zur Anzeige von Gleichzeitigkeit mit Gegenwartsbezug, der in Opposition zum Vergangenheitsbezug von als steht.

Die Duden-Erklärungen zu als und wie wirken auf mich noch unbefriedigend. Erkennbar ordnet sich wie in das Inventar der deutschen $w$-Indefinita ein, zu denen auch wenn gehört und das den Inventaren der kontiguitätsrelationalen $d$ Deiktika und der similaritätsrelationalen $s$-Deiktika, zu denen als gehört, gegenübersteht. Indefinita sind nicht-deiktisch, d.h. ihnen fehlt die Rückbindung an den Sprecher über einen Diskursanker. Anders gesagt, sie kodieren reine R/ERelationen. In ihren Formeln kommen die Relate $\mathrm{O}$ und $\mathrm{S}$ nicht vor. Dieses Nicht-Vorkommen schließt allerdings nicht aus, dass Relationen zu O und S im Kontext ergänzt werden. Genau das geschieht bei wie in (56), wo das Präteritum Distanz von R zu S und O anzeigt. Auch in (57) ist, anders als die DudenAutoren es andeuten, eine solche Distanz-Lesart per Inferenz zu bevorzugen, und zwar in narrativen Kontexten über die Kohärenz der Fiktionalität, genau wie bei als in (55).

Andererseits erlaubt temporales wie durchaus eine echte Gegenwarts-Lesart, etwa wenn man mit Bezug auf das gerade stattfindende Sprechereignis sagt:

(58) Wie ich das sage, fällt mir ein, dass ich Petra anrufen muss.

oder auch:

(59) Wo ich das sage, fällt mir ein, dass ich Petra anrufen muss.

nicht aber in gleicher Bedeutung:

(60) Als ich das sage, fällt mir ein, dass ich Petra anrufen muss.

Wie legt also bloß die Relation zwischen E und R fest, und zwar ebenso wie als, $d a$ und so auf Nähe (Gleichzeitigkeit bzw. Überlappung). Die entsprechende Relationsformel, die übrigens auch für wo gilt, lautet R,E. Wie wir bereits gesehen haben, lässt wenn im Unterschied dazu diese Relation unbestimmt.

Wir können also das Konnektoren-Diagramm aus Figur (50) abermals erweitern $\mathrm{zu}$ : 
(61)

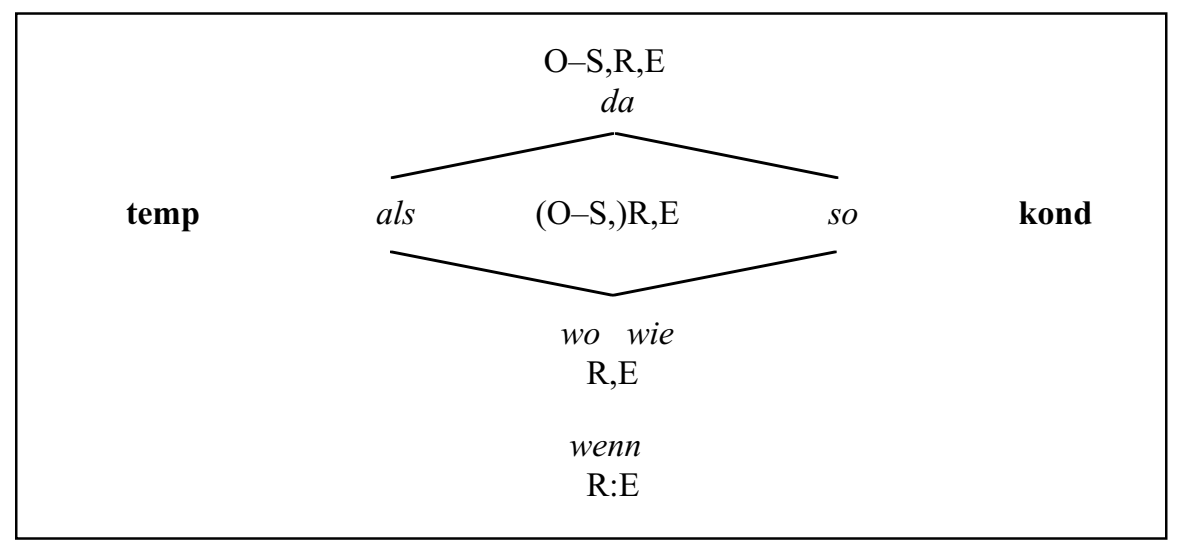

Hier sind wo und wenn kontiguitätsrelationale Konnektoren, wie dagegen ist von Haus aus, wie so und als, similaritätsrelational. Es wird aber, ebenso wie diese, auch zur Anzeige von Kontiguitätsrelationen verwendet.

Die Opposition zwischen als und wie hat also, anders als die Duden-Autoren meinen, mit Temporalität nichts zu tun. Sie betrifft nicht die Vorzeitigkeit vs. Nicht-Nachzeitigkeit des Bezugssachverhaltes zum Sprechereignis, sondern sie betrifft die Kontextabhängigkeit. Als ist ein schwaches Deiktikum, wie ist nichtdeiktisch. ${ }^{12}$ Dass Nachzeitigkeits-Lesarten bei wie ausgeschlossen sind, erklärt sich nicht semantisch, sondern aus ihrer pragmatischen Unplausibilität, denn Gleichzeitigkeit bzw. Überlappung des situierten Sachverhaltes mit einem zukünftigen Bezugssachverhalt können vom Sprechzeitpunkt aus nicht beurteilt werden. Daher wird für Zukunfts-Aussagen semantisch weniger festlegendes wenn verwendet:

(62) Wenn ich das sage, wird mir einfallen, dass ich Petra anrufen muss.

\footnotetext{
${ }^{12}$ Wie und wo können dennoch auch anaphorisch verwendet werden. So lautet der in (48) zitierte Anfang des Märchens Der Froschkönig im Original: In den alten Zeiten, wo das Wünschen noch geholfen hat (...). Das anaphorisch gebrauchte Indefinitum lässt offen, ob und wann der genannte Sachverhalt außerhalb des Textes faktisch bestanden haben mag. Während als aufgrund seiner deiktischen Semantik Annahmen über einen Referenzbereich außerhalb des Textes nahelegt, errichten anaphorische Indefinita rein textinterne Wiederaufnahmebeziehungen. Die Brüder Grimm haben hier also sehr zu Recht wo und nicht als als Konnektor gewählt.
} 


\subsection{Syntagmatische Interaktion}

Nach diesen paradigmatischen Beobachtungen zur Semantik von als möchte ich nun abschließend die entwickelte Relations-Formel anhand eines syntagmatischen Phänomens testen, das Zifonun et al. (1997: 2325f.) als "peripheren Temporalsatz" bezeichnen (siehe auch oben, Beispiel (1)):

(63) Ich stand gerade an der Haustür, als plötzlich das Gewitter einsetzte.

Die Autoren der Grammatik der deutschen Sprache (GDS) nehmen an, dass in solchen Fällen der als-Satz nicht "in der üblichen Weise" die "Betrachtzeit für den Obersatz" spezifiziert. Vielmehr werde "die Überlappung zwischen einem noch andauernden Ereignis (Hauptsatz-Denotat) und einem neu einsetzenden Ereignis (Nebensatz-Denotat)" signalisiert (ebd.: 2325). In meine Variante des Reichenbach-Modells übersetzt, entspricht das der These, dass bei solchen alsSätzen die Anordnung von Bezugssachverhalt (R) und situiertem Sachverhalt (E) umgekehrt sei, der Nebensatz mit als also den situierten Sachverhalt einführe und der Obersatz den Bezugssachverhalt bereitstelle. Entsprechend paraphrasiert die GDS den als-Satz in (63) durch Da setzte plötzlich das Gewitter ein (ebd.: 2326). Eine solche Paraphrase ist mit meiner semantischen Analyse von $d a$ und als nicht kompatibel. Wenn meine Analyse richtig ist, dann muss entweder (63) anders paraphrasiert werden, oder das als in "peripheren Temporalsätzen" unterscheidet sich in seiner abstrakten Relationsbedeutung von den bisher behandelten als-Varianten und von der üblichen Semantik aller subordinierenden Konjunktionen. Warum und wie es zu einer solchen Abweichung kommen könnte, erklären die GDS-Autoren nicht.

Ich möchte dieser Frage im folgenden detailliert nachgehen und dafür argumentieren, dass es nur eine einzige unterordnende Konjunktion als gibt und dass gerade die von mir gegebene Bedeutungsbeschreibung ihr scheinbar ungewöhnliches Verhalten in Sätzen wie (63) nicht nur beschreiben, sondern auch erklären kann. Dazu werde ich annehmen, dass die Konjunktion als sich in allen Fällen so verhält wie ein prototypischer transitiver Konnektor und als Komplement einen Ausdruck nimmt, der das Bezugsobjekt bezeichnet. Es gibt also keinen zusätzlichen Erklärungsbedarf für eine Umordnungs-Annahme. Die von den GDS-Autoren (ebd.: 2323) beschriebenen allgemeinen Eigenschaften "peripherer Satzadverbialsätze" ergeben sich meiner Auffassung nach im wesentlichen aus der relationalen Semantik der dabei zum Einsatz kommenden Konnektoren und ihrem syntagmatischen Zusammenspiel mit weiteren Regeln, die unabhängig von der Konnektorensemantik motiviert sind.

Um Sätze wie (63) verstehen zu können, muss man mindestens vier linguistischen Oppositionen berücksichtigen: 
1. Rahmen vs. Figur (ground vs. figure; cf. Klein 1974: 81 f.; Wallace 1982). Diese psychologisch-rhetorische Opposition regelt das Arrangement der besprochenen Sachverhalte auf der Bühne unserer Vorstellung. Rahmen-Sachverhalte bilden die Kulisse, Figur-Sachverhalte finden gleichsam an der Rampe statt, sind also in der Vorstellung prominent. In (63) ist der Rahmen durch den Sachverhalt ich stand an der Haustür gegeben, während plötzlich setzte das Gewitter ein Figur ist. Sprachlich wird der Rahmen meist durch imperfektive Aspektualität, die Figur durch perfektive Aspektualität markiert.

2. Bezugsobjekt vs. situiertes Objekt (R vs. E; cf. Reichenbach 1947=1999: 273ff.; landmark vs. trajector; cf. Langacker 1987: 231ff.). Diese semantische Opposition dient zur Vernetzung der Objekte im Diskurs. Der Sprecher ist weitgehend frei in der Entscheidung, welche Objekte er situieren und relativ zu welchen anderen Objekten er sie situieren möchte. Dabei spielen aber auch psychologische Faktoren eine Rolle, die der subjektiven Kontrolle entzogen sind, etwa Salienz (cf. Svorou 1994: 8ff.). Syntaktisch sind Ausdrücke für Bezugsobjekte gewöhnlich Komplemente von Funktoren (z. B. Konnektoren). Ausdrücke für situierte Objekte sind in Bezug auf diese Funktoren gewöhnlich frei. In (63) ist also plötzlich setzte das Gewitter ein der Bezugssachverhalt und ich stand an der Haustür der situierte Sachverhalt.

3. Hintergrund vs. Fokus (cf. Jacobs 1984: 28ff.). Diese rhetorische Opposition regelt die Verteilung von vorausgesetzter und neuer Information im Diskurs. Der Fokus wird intonatorisch markiert, und zwar durch den Hauptakzent des Satzes. Trägt von zwei Teilsätzen nur einer einen Hauptakzent und bleibt der andere unbetont oder trägt einen Nebenakzent, so wird der vom akzentuierten Teilsatz bezeichnete Sachverhalt als Informationsfokus (neue Information), der andere als Informationshintergrund (vorausgesetzte Information) verstanden. Da die GDS-Autoren ihre Beispielsätze ohne Intonation notieren, ist nicht klar, wo in (63) der Fokus liegt. Es sind zwei Varianten möglich: ${ }^{13}$

(63.a) Ich stand gerade an der /HAUStür, als plötzlich das GeWIT\ter einsetzte.

(63.b) Ich stand gerade an der /HAUS \tür, als plötzlich das Gewitter einsetzte.

\footnotetext{
${ }^{13}$ Ich markiere Haupt- und Nebenakzent durch Großbuchstaben. Vor der Silbe mit Nebenakzent steht ein steigender, nach der Silbe mit Hauptakzent ein fallender Schrägstrich. Gibt es nur eine Silbe mit Satzakzent, so steht der steigende Schrägstrich vor und der fallende nach dieser Silbe. Einfachheitshalber arbeite ich im folgenden durchweg mit der Annahme nur einer Intonationsgruppe.
} 
Dem von den GDS-Autoren diskutierten "peripheren Temporalsatz" dürfte (63.a) mit fokussiertem als-Satz entsprechen. Hier ist das Einsetzen des Gewitters ein selbstständig assertierter Sachverhalt, wie ihn die Autoren (ebd.: 2323) im Sinn haben. In (63.b) bleibt der als-Satz dagegen unbetont und wird infolgedessen als Träger vorausgesetzter Information (Hintergrund) interpretiert. Die neue Information liegt im Obersatz.

4. Vordersatz vs. Nachsatz (cf. Duden 1998: 831). Diese syntaktische Opposition betrifft die serielle Abfolge der Teilsätze. Ich bezeichne als Vordersatz den zuerst serialisierten und als Nachsatz den anschließend serialisierten Teilsatz. Aufgrund der Untersuchungen zur Funktionalen Satzperspektive bzw. Thema-Rhema-Gliederung (cf. Heidolph et al. 1984: 740f.; Eroms 1986: 59f.) ist bekannt, dass der Satzakzent im Deutschen zum Satzende tendiert, also bevorzugt auf den Nachsatz fällt, während der Vordersatz eher unakzentuiert bleibt oder nur einen Nebenakzent erhält, also den Hintergrund bereitstellt.

Es ist deutlich, dass in als-Sätzen auch Rahmen vs. Figur und Bezugsobjekt vs. situiertes Objekt sich bruchlos in den Vordersatz-Hintergrund- und NachsatzFokus-Parallelismus einordnen. Denn als verlangt, wie wir gesehen haben, dass der Bezugssachverhalt spezifisch und kategorial erwartbar ist. Damit aber ist er prädestiniert für die Hintergrundrolle, während sich der zu situierende Sachverhalt für die Fokusrolle aufdrängt. Abweichungen von dieser durch die Semantik von als geforderten Zuordnung bedürfen besonderer Rechtfertigung. In einem prototypischen, unauffälligen als-Gefüge fallen daher Rahmen, Bezugssachverhalt, Hintergrund und Vordersatz einerseits sowie Figur, situierter Sachverhalt, Fokus und Nachsatz andererseits zusammen:

(64.a) Als ich gerade an der /HAUStür stand, setzte plötzlich das GeWIT\ter ein.

In dieser Anordnung passen die durch als angezeigte Sachverhaltsrelation und die psychologisch-rhetorische Informationsstruktur optimal zusammen.

Betrachten wir nun nacheinander verschiedene Abweichungen von der Idealanordnung. Nur leicht markiert ist die Umordnung von Figur und Rahmen:

(64.b) Als plötzlich das Ge/WITter einsetzte, stand ich gerade an der HAUS \tür.

Hier fallen weiterhin Bezugssachverhalt, Hintergrund und Vordersatz sowie situierter Sachverhalt, Fokus und Nachsatz zusammen. Dagegen schwächt die Vertauschung von Figur und Rahmen den Kontrast dieser Opposition ab. Das Einsetzen des Gewitters wird dadurch wie in der Zeitlupe gedehnt, das Stehen an der Haustür umgekehrt gerafft. Die beiden, aspektuell ungleichartigen Sachverhalte werden rhetorisch synchronisiert.

Stärker markiert ist der Positionstausch von E und R, wie in (63.a), hier als (64.c) wiederholt: 
(64.c) Ich stand gerade an der /HAUStür, als plötzlich das GeWIT \ter einsetzte.

Die Fokussierung des Bezugssachverhaltes ist mit der Semantik von als nur dann vereinbar, wenn der Bezugssachverhalt kategorial erwartbar bleibt. Das bedeutet, dass Information fokussiert wird, die nicht völlig neu sein darf. Im übrigen fallen aber ganz regelkonform Fokus, Figur und Nachsatz sowie Hintergrund, Rahmen und Vordersatz zusammen. Diese Analyse unterscheidet sich klar von der der GDS (2325f.), aber sie macht zugleich deutlich, woher die Intuition rührt, dass in "peripheren Temporalsätzen" E und R vertauscht sind. Sie sind in der Tat vertauscht, jedoch nicht in Bezug auf die Konjunktion als, sondern in Bezug auf Hintergrund/Rahmen/Vordersatz und Fokus/Figur/Nachsatz. Sätze wie (64.c) schaffen zur semantischen Abstufung zwischen E und R ein informationsstrukturelles Gegengewicht, das die Arbeitsteilung der beiden Sachverhalte bei der Situierung verwischt. Während in der Idealanordnung (64.a) eindeutig einer der Sachverhalte situiert wird und der andere ihn situiert, scheinen sich in (64.c) die Sachverhalte gegenseitig zu situieren. Das erklärt die von den GDS-Autoren (2323) beobachtete Ähnlichkeit zwischen Sätzen mit "peripheren Satzadverbialsätzen" und "koordinativen Reihen von Hauptsätzen". Der Positionstausch von E und R erhöht die narrative Dynamik.

Abermals stärker markiert ist der gleichzeitige Positionstausch von $\mathrm{E}$ und $\mathrm{R}$, Figur und Rahmen sowie Fokus und Hintergrund, oder, wie man auch sagen könnte, der Austausch von Vorder- und Nachsatz:

(64.d) Plötzlich setzte das Ge/WIT \ter ein, als ich gerade an der Haustür stand.

Gegenüber (64.a) ist hier bloß die Reihenfolge der Teilsätze umgekehrt. Alles andere bleibt gleich, d.h. fokussiert ist der erste Teilsatz (der Obersatz), der E und Figur bezeichnet, während der zweite (der Nebensatz), der R und Rahmen bezeichnet, unakzentuiert bleibt. Dieser Vorgang ist aus der Literatur zur Funktionalen Satzperspektive als Topikalisierung des Rhemas bekannt (cf. Eroms 1986: 69) und bewirkt dessen (emphatische) Hervorhebung, hier also eine Hervorhebung des situierten Sachverhaltes E, der zugleich Figur ist.

In (64.b) haben wir gesehen, dass Figur und Rahmen relativ leicht ausgetauscht werden können. Dabei ergibt sich bloß ein leichter Neutralisierungseffekt in der Aspektualität. Die gleiche Vertauschung können wir auf (64.d) anwenden und erhalten dann die zweite Intonations-Variante von (63), die ebenfalls den schon beschriebenen Zeitlupeneffekt bezüglich des Einsetzen des Gewitters und den Zeitraffereffekt bezüglich des Stehens an der Haustür aufweist:

(64.e) Ich stand gerade an der /HAUS \tür, als plötzlich das Gewitter einsetzte.

Im Vergleich mit (64.b) wirkt (64.e) allerdings deutlich motivierungsbedürftiger, denn hier wird zwar, wie es die Semantik von als verlangt, der situierte Sachverhalt $\mathrm{E}$ fokussiert, während der Bezugssachverhalt den Hintergrund bildet, aber 
der Fokus ist topikalisiert, und das obgleich er auf den Rahmen-Sachverhalt fällt, während der Figur-Sachverhalt im defokussierten Nachsatz steht. Diese Konstellation kann vor allem verwendet werden, um einen Kontrastakzent auf E zu legen, etwa wenn man eine anderslautende Behauptung richtigstellen möchte.

Die bis hierher besprochenen Abweichungen von der Idealanordnung sind übliche stilistische Varianten, die zur Hervorbringung gut beschreibbarer Effekte genutzt werden. Im folgenden werde ich noch auf drei weitere Abweichungen eingehen, die zunehmend markierter werden, weil sie nur schwer motivierbar erscheinen. Auch solche Varianten werfen ein erhellendes Licht auf die Semantik von als.

Beginnen wir mit dem gleichzeitigen Austausch von Figur und Rahmen sowie von $\mathrm{E}$ und $\mathrm{R}$ :

(64.f) Plötzlich setzte das Ge/WITter ein, als ich gerade an der HAUS \tür stand.

Diese Variante widerspricht der relationalen Semantik von als, da der situierte Sachverhalt E im Hintergrund steht und der Bezugssachverhalt R fokussiert ist. Es wird also Information fokussiert, die nicht neu sein darf. Wie bei (64.c) steht der Fokus regelkonform im Nachsatz, aber anders als in (64.c) fällt er nicht auf den Figur-, sondern auf den Rahmen-Sachverhalt. Deshalb erhöht (64.f) nicht wie (64.c) die narrative Dynamik. Beide Teilsätze von (64.f) enthalten zwei regelkonforme Merkmalpaare, die untereinander nur schwer verträglich sind: Figur/E vs. Hintergrund/Vordersatz und Rahmen/R vs. Fokus/Nachsatz. Es hängt stark vom Kontext $\mathrm{ab}$, ob sich für eine solche Anordnung eine geeignete Motivation finden lässt. In den meisten Kontexten dürfte (64.f) stilistisch unbeholfen wirken.

Eine sehr starke stilistische Markierung produziert der Positionstausch von Fokus und Hintergrund, wenn alle übrigen Eigenschaften gleich bleiben:

(64.g) Als ich gerade an der /HAUS \tür stand, setzte plötzlich das Gewitter ein.

In (64.e) haben wir gesehen, dass die Fokussierung des Vordersatzes, wenn dort der Teilsatz steht, der den Rahmen-Sachverhalt bezeichnet, als Kontrastakzent interpretiert werden kann, um anderslautende Behauptungen richtigzustellen. Dies ist bereits eine relativ stark markierte Anordnung, die jedoch dadurch akzeptabel wird, dass die Konjunktion als regelkonform den HintergrundSachverhalt einführt. Wenn diese Eigenschaft wie in (64.g) wegfällt, weil als in den Vordersatz gestellt und der in dieser Position fokussierte RahmenSachverhalt damit zum Bezugssachverhalt für die Situierung wird, erhöht sich die stilistische Markierung weiter. Es wird nun ein Kontrastakzent auf den Bezugssachverhalt gelegt, also auf Information, die nicht neu sein darf. Rein sprachlich ist das zwar noch möglich, aber stilistisch sind für solche Mitteilungsabsichten andere Mittel, etwa Spaltsatzkonstruktionen, zu bevorzugen. 
Die letzte Variante schließlich vertauscht gegenüber (64.g) zusätzlich noch Figur und Rahmen:

(64.h) Als plötzlich das Ge/WIT\ter einsetzte, stand ich gerade an der Haustür.

Auch hier treffen im Vorder- und im Nachsatz je zwei regelkonforme Merkmalpaare aufeinander, die miteinander unverträglich sind: R/Vordersatz vs. Figur/Fokus und E/Nachsatz vs. Rahmen/Hintergrund. Die wechselseitige Unverträglichkeit dieser Paare ist aber viel stärker als die in (64.f). Zum einen wird die als-Regel verletzt, nach der E fokussiert und R den Hintergrund bilden soll. Zum anderen wird die Regel der Informationsverteilung verletzt, nach der der Nachsatz fokussiert und der Vordersatz im Hintergrund stehen soll. Da außerdem auch noch $\mathrm{E}$ und $\mathrm{R}$ in markierter Anordnung zu Figur und Rahmen stehen, so dass die Aspektualität und die Situierungsverhältnisse unklar bleiben, kann diese Variante wohl nur noch als maximal dysfunktional und daher stilistisch inakzeptabel eingestuft werden. Sie ist nicht einmal mehr für Kontrastakzentuierungen brauchbar.

\section{$6 \quad$ Schluss}

Im vorliegenden Aufsatz bin ich, frei nach Harald Weydt, der Frage nachgegangen, warum die temporale Konjunktion als 'als' heißt, und was sie bedeutet. $\mathrm{Zu}$ diesem Zweck habe ich ein umfassendes Beschreibungsmodell für die Konnektorensemantik entworfen und einige ausgewählte Konnektoren, die zu als in Opposition stehen, in diesem Rahmen behandelt.

Warum als 'als' heißt, lässt sich zunächst scheinbar einfach formgeschichtlich erklären. Da aber gerade bei stark grammatikalisierten Funktionswörtern frühere Entwicklungsstadien subtile semantische Spuren zu hinterlassen pflegen, ist die Formgeschichte nur der Einstieg in die Frage nach der gegenwärtigen Bedeutung.

Die Konjunktion als bedeutet nach der hier vorgeschlagenen Analyse:

In zeitlicher Überlappung mit dem spezifischen, faktischen und im Kontext unabhängig vom Sprecher kategorial erwartbaren Bezugssachverhalt $\mathbf{R}$ findet der zu situierende Sachverhalt $\mathbf{E}$ statt.

Der relationale Kern dieser Paraphrase wird durch die Formel (O-S,)R,E wiedergegeben.

Die Konnektoren, die ich zusammen mit als behandelt habe, zerfallen in drei Gruppen: $d$-Deiktika, $s$-Deiktika und $w$-Indefinita. Die $d$-Deiktika, für die exemplarisch $d a$ und dann behandelt wurden, sind Definita und kodieren Kontiguitätsrelationen. An ihnen wurde die Systematik der vier relationalen Domänen (Raum, Zeit, Alethik/Episteme, Deontik) und der drei relationalen Perspektiven (Situierung, Bedingung, Verursachung) erläutert. Die $s$-Deiktika kodieren von 
Haus aus Similaritätsrelationen, haben ihren Anwendungsbereich aber auf Kontiguitätsrelationen ausgedehnt, und sind dabei schwachdeiktisch geworden. $\mathrm{Zu}$ dieser Gruppe gehören so, also und als, die zugleich eine etymologische Entwicklungskette bilden und die sprachhistorische Ausdifferenzierung eines Teilinventars von Funktionselementen veranschaulichen. Die $w$-Indefinita können Kontiguitätsrelationen (wenn) oder Similaritätsrelationen (wie) kodieren. Letztere können wiederum auf Kontiguitätsrelationen ausgedehnt werden.

Drei der im Detail behandelten Konnektoren ( $d a$, so und als) kommen in intransitiver (komplementloser) und transitiver (komplementbedürftiger) Verwendung vor, zwei (wie und wenn) werden nur transitiv verwendet, zwei andere (dann und also) nur intransitiv. Die intransitiven Konnektoren sind syntaktische Konstituenten des Sprachausdrucks, der die zu situierende bzw. zu beschreibende Entität bezeichnet, während die transitiven Konnektoren den Sprachausdruck, der die Bezugsentität bezeichnet, als Komplement nehmen.

Unter den intransitiven Konnektoren (Adverbien) haben wir $d a$ als universalen, domänenübergreifenden Situierungsanzeiger und so als Similaritätsanzeiger kennengelernt. Neben $d a$ steht in der temporalen, alethisch-epistemischen und deontischen Domäne dann, das zur Anzeige von Bedingungs- und Verursachungsrelationen bevorzugt wird. So ist bei Übertragung auf Kontiguitätsrelationen vor allem auf die alethisch-epistemische und die deontische Domäne, mit Einschränkungen auch auf die temporale Domäne anwendbar, nicht dagegen auf die Raumdomäne. In der alethisch-epistemischen und der deontischen Domäne konkurriert es mit also, das sich auf Schlussfolgerungsrelationen spezialisiert hat. In der Zeitdomäne, besonders bei der zeitlichen Situierung, konkurriert es mit intransitivem als, das allerdings in der Gegenwartssprache eher als temporaler Quantor fungiert. Wir können diese funktionale Verteilung durch folgendes Übersichtsschema verdeutlichen: 


\begin{tabular}{|c|c|c|c|c|}
\hline Intransitiva & Raum & Zeit & Alethik/Episteme & Deontik \\
\hline Beschreibung & & so & & \\
\hline Situierung & $d a$ & 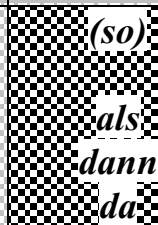 & 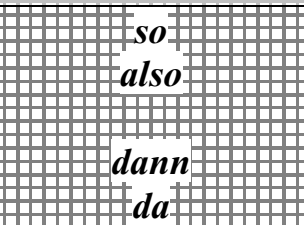 & 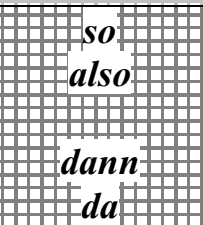 \\
\hline Bedingung & & $33^{\text {(als })}$ & 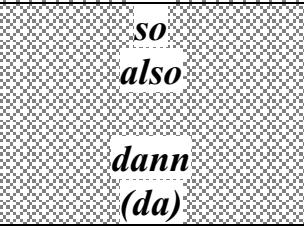 & 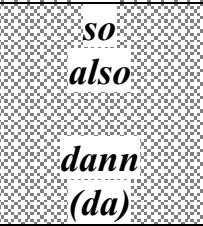 \\
\hline Verursachung & & $33+$ dann & 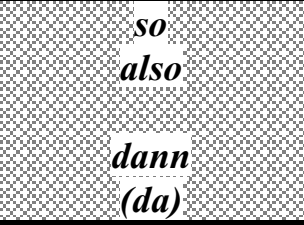 & 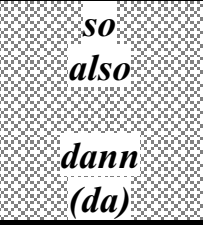 \\
\hline
\end{tabular}

Die Hinterlegung der Felder mit abgestuften Grautönen deutet an, für welche Bereiche des Schemas jeweils gleiche Konnektor-Inventare Verwendung finden.

Unter den transitiven Konnektoren (Konjunktionen) zeigt $d a$ vor allem alethischepistemische und deontische Relationen an. Zur Anzeige von Zeit- und Raumrelationen ist es in der Gegenwartssprache kaum noch üblich. Wie dient allgemein zur Anzeige von Similaritätsrelationen. Im Kontiguitätsbereich ist es auf temporale Situierungsbeziehungen beschränkt. Transitives so ist gegenwartssprachlich selten. Es dient zur Anzeige temporaler, alethisch-epistemischer und deontischer Bedingungen und Ursachen. Im allgemeinen wird für diese Aufgaben eher wenn verwendet, das auch zeitliche, alethisch-epistemische und deontische Situierungen anzeigen kann. Transitives als ist auf die Anzeige zeitlicher Situierungen beschränkt: 


\begin{tabular}{|c|c|c|c|c|}
\hline Transitiva & Raum & Zeit & $\begin{array}{l}\text { Alethik/ } \\
\text { Episteme }\end{array}$ & Deontik \\
\hline Beschreibung & wie & wie & wie & wie \\
\hline Situierung & (da) & $\left\{\begin{array}{l}\text { (da) } \\
\text { als } \\
\text { wien }\end{array}\right.$ & 央 $d a^{7}$ & 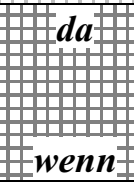 \\
\hline Bedingung & & $\begin{array}{l}(d a) \\
\text { wenn } \\
\text { so }\end{array}$ & $\begin{array}{c}d a \\
\text { wenn } \\
\text { so }\end{array}$ & $\begin{array}{l}d \mathrm{da} \\
\text { wenn } \\
\text { so } \\
\text { so }\end{array}$ \\
\hline Verursachung & & $(d a)$ & $\begin{array}{l}\text { da } \\
\text { wenn } \\
\text { so }\end{array}$ & 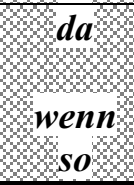 \\
\hline
\end{tabular}

Die spezifische These dieses Aufsatzes besagt, dass $d a$, so, also und als die gleiche abstrakt-relationale Grundbedeutung haben, die durch die Formel $\mathrm{O}-\mathrm{S}, \mathrm{R}, \mathrm{E}$ wiedergegeben wird. Die semantischen Oppositionen zwischen ihnen ergeben sich aus einer dreifach gestaffelten Definitheitsskala und aus den relationalen Domänen und Perspektiven. Die Definitheitsskala betrifft die semantische Rückbindung an den Äußerungskontext und an den Sprecher. Bei den $d$ Deiktika ist diese voll vorhanden, bei den transitiven $s$-Deiktika nur abgeschwächt (was durch Klammerung der Teilformel O-S ausgedrückt wird), bei den $w$-Indefinita gar nicht. Bei letzteren kann eine kontextuelle Rückbindung nur pragmatisch inferiert werden. In Bezug auf die Konjunktion als hat die Diskussion meiner Auffassung nach gezeigt, dass ihre semantische Beschreibung durch die Relationsformel $(\mathrm{O}-\mathrm{S},) \mathrm{R}, \mathrm{E}$ ein geeignetes Instrument ist, um ihre paradigmatischen Oppositionen im Konnektorensystem zu beschreiben und um ihr syntagmatisches Verhalten im Zusammenspiel mit Fokussierung, Serialisierung, Aspektualität und Temporalität zu erklären.

Zugleich gibt die These von der Bedeutungsgleichheit von so, also und als auch einen Hinweis darauf, warum als mit Kontiguitäts-Semantik (von allen anderen als-Varianten, die bis heute reine Similaritäts-Semantik besitzen, wird hier abgesehen) auf temporale Relationen beschränkt ist. Offenbar hat die sprachgeschichtliche Ausweitung des Anwendungsbereiches von so und insbesondere die dadurch neu aufgekommene Opposition zwischen $d$ - und $s$-Deixis in der Kontiguitäts-Semantik Bedarf für eine Erweiterung des Inventars $s$-deiktischer Konnektoren für die Kodierung von Kontiguitätsrelationen entstehen lassen. Der 
Übergang von Similarität zu Kontiguität vollzog sich zuerst in den abstrakteren Domänen der Alethik/Episteme und Deontik. Hier blieb verstärktes allso, das ansonsten zur Zeit Adelungs aus der Mode kam (cf. Paul 1992: 25), in der Form des neuen Kontiguitätskonnektors also erhalten. $\mathrm{Zu}$ beschreibendem und schlussfolgerndem also trat formal gekürztes als in Opposition, indem es die temporale Relationsdomäne besetzte. Dieser Vorgang ging wahrscheinlich mit einer alternativen Interpretation der verblassenden morphologischen Komponente all- einher, die also und als gemeinsam ist. Bei also diente sie als Verstärkungselement, bei als dagegen als temporaler Quantor, wie wir ihn im adverbialen als noch heute im südwestdeutschen Sprachraum finden. ${ }^{14}$ In den gegenwartssprachlichen Konnektoren also und als sind beide Interpretationen von all-freilich längst unkenntlich geworden.

Auch nach der Transitivierung von $d a$ und so bleibt eine klare Opposition zwischen so und als erhalten, die nun aber nicht mehr zwischen den Domänen, sondern zwischen den Perspektiven verläuft. Die deutsche Konjunktion als ist, anders als ihr englisches Gegenstück as, ein rein situierender Konnektor geblieben, während die konditionalen und kausalen Perspektiven im Deutschen von $d a$, so und wenn (mit entsprechender Definitheitsabstufung) übernommen wurden.

\section{Literaturangaben}

Anderson, Robert R. et al. (ed.) (1989): Frühneuhochdeutsches Wörterbuch. Band 1. Berlin.

Bierwisch, Manfred (1988): "On the grammar of local prepositions". In: Bierwisch, Manfred et al. (ed.): Syntax, Semantik und Lexikon. Rudolf Ruzicka zum 65. Geburtstag. Berlin: 1-65.

Blühdorn, Hardarik (1993): Funktionale Zeichentheorie und deskriptive Linguistik. Ein Entwurf am Beispiel des Gegenwartsdeutschen. Erlangen.

Blühdorn, Hardarik (im Druck): "Rauminformation und Demonstrativität. Am Beispiel des Deutschen". Deutsche Sprache 30, 2002.

Brecht, Bertolt (1967): "Die Straßenszene. Grundmodell einer Szene des epischen Theaters". In: Brecht, Bertolt: Werkausgabe Edition Suhrkamp, Gesammelte Werke in 20 Bänden. Band 16: Schriften zum Theater 2. Frankfurt/Main: 546-558.

Bublitz, Wolfram (1977): "Deutsch 'aber' als Konjunktion und als Modalpartikel". In: Sprengel, Konrad et al. (ed.): Semantik und Pragmatik. Akten des 11. Linguistischen Kolloquiums Aachen 1976. Band 2. Tübingen: 199-209.

Bublitz, Wolfram (1978): Ausdrucksweisen der Sprechereinstellung im Deutschen und Englischen. Untersuchungen zur Syntax, Semantik und Pragmatik der deutschen Modalpartikeln und Vergewisserungsfragen und ihrer englischen Entsprechungen. Tübingen.

${ }^{14}$ Diese These deutet darauf hin, dass die beiden bei Kluge (1975: 16) getrennt lemmatisierten Varianten von als letztlich vielleicht doch etymologisch zusammengehören. 
Burkhardt, Armin (1987): "SOSO? Kritik und weiterführende Überlegungen zu Konrad Ehlichs Aufsatz über die Funktionen des deutschen so." In: Rosengren, Inger (ed.): Sprache und Pragmatik. Lunder Symposium 1986. Stockholm: 299-313.

Diessel, Holger (1996): "German 'wenn': A study in cognitive semantics". Buffalo Papers in Linguistics 96-01: Proceedings from the Second Buffalo-Toronto Student Conference in Linguistics. Buffalo.

Diewald, Gabriele M. (1999): Die Modalverben im Deutschen. Grammatikalisierung und Polyfunktionalität. Tübingen.

Di Meola, Claudio (2000): Die Grammatikalisierung deutscher Präpositionen. Tübingen.

Duden (1998): Grammatik der deutschen Gegenwartssprache. 6. Aufl. Mannheim.

Duden (2001): Deutsches Universalwörterbuch. 4. Aufl. Mannheim.

Ehlich, Konrad (1987): "so - Überlegungen zum Verhältnis sprachlicher Formen und sprachlichen Handelns, allgemein und an einem widerspenstigen Beispiel". In: Rosengren, Inger (ed.): Sprache und Pragmatik. Lunder Symposium 1986. Stockholm: 279-298.

Ehrich, Veronika (1982): "Da and the System of Spatial Deixis in German". In: Weissenborn, Jürgen/Klein, Wolfgang (ed.): Here and There. Cross-linguistic Studies on Deixis and Demonstration. Amsterdam: 43-63.

Ehrich, Veronika (1992): Hier und Jetzt. Studien zur lokalen und temporalen Deixis im Deutschen. Tübingen.

Eroms, Hans-Werner (1986): Funktionale Satzperspektive. Tübingen.

Frawley, William (1992): Linguistic Semantics. Hillsdale.

Freksa, Christian et al. (ed.) (1998): Spatial Cognition. An Interdisciplinary Approach to Representing and Processing Spatial Knowledge. Berlin.

Freksa, Christian et al. (ed.) (2000): Spatial Cognition II. Integrating Abstract Theories, Empirical Studies, Formal Methods, and Practical Applications. Berlin.

GDS = Zifonun, Gisela et al. (1997): Grammatik der Deutschen Sprache. Berlin.

Hahnemann, Suzan (1999): Vergleiche im Vergleich. Zur Syntax und Semantik ausgewählter Vergleichsstrukturen mit 'als' und 'wie' im Deutschen. Tübingen.

Hartweg, Frédéric/Wegera, Klaus-Peter (1989): Frühneuhochdeutsch. Eine Einführung in die deutsche Sprache des Spätmittelalters und der frühen Neuzeit. Tübingen.

Heidolph, Karl Ehrich et al. (1984): Grundzüge einer deutschen Grammatik. 2. Aufl. Berlin.

Hentschel, Elke/Weydt, Harald (1983): "Der pragmatische Mechanismus: denn und eigentlich". In: Weydt, Harald (ed.): Partikeln und Interaktion. Tübingen: 263-273.

Hentschel, Elke/Weydt, Harald (1990): Handbuch der deutschen Grammatik. Berlin.

Jacobs, Joachim (1984): "Funktionale Satzperspektive und Illokutionssemantik". Linguistische Berichte 91: 25-58.

Jakobson, Roman ([1956=] 1971): "Two Aspects of Language and Two Types of Aphasic Disturbances". In: Jakobson, Roman/Halle, Morris. Fundamentals of Language. 2nd edition. The Hague: 67-96.

Klein, Horst G. (1974): Tempus, Aspekt, Aktionsart. Tübingen.

Kluge, Friedrich (1975): Etymologisches Wörterbuch der deutschen Sprache. 21. Aufl. Berlin. 
Lakoff, George (1987): Women, Fire, and Dangerous Things. What Categories Reveal about the Mind. Chicago.

Langacker, Ronald W. (1987): Foundations of Cognitive Grammar. Volume 1: Theoretical Prerequisites. Stanford.

Lehmann, Christian (1995): Thoughts on Grammaticalization. Revised and expanded version. München.

Lyons, John (1977): Semantics. 2 Bde., Cambridge.

Moilanen, Markku (1979): "Zur pragmatischen Funktion der Demonstrativadverbien hier, da und dort". In: Weydt, Harald (ed.): Die Partikeln der deutschen Sprache. Berlin: $187-200$,

Paul, Hermann (1992): Deutsche Wörterbuch. 9. Aufl. Tübingen.

Rauh, Gisa (1983): "Aspects of Deixis". In: Rauh, Gisa (ed.): Essays on Deixis. Tübingen: 9-60,.

Redder, Angelika (1987): "wenn..., so. Zur Korrelatfunktion von so". In: Rosengren, Inger (ed.): Sprache und Pragmatik. Lunder Symposium 1986. Stockholm: 315-326,.

Redder, Angelika (1990): Grammatiktheorie und sprachliches Handeln: "denn" und

"da". Tübingen.

Reichenbach, Hans ([1947=]1999): Grundzüge der symbolischen Logik (Elements of Symbolic Logic). Gesammelte Werke, Bd 6. Deutsch von Matthias Varga von Kibéd, Claudia Thomé und Stephan Bayerl. Braunschweig.

Rünneburger, Henri (1998): "Elsässisch 'àls': Signifikant mit mehrfachem Signifikat Lexem oder Partikel?" In: Dalmas, Martine / Sauter, Roger (ed.): Grenzsteine und Wegweiser. Textgestaltung, Redesteuerung und formale Zwänge. Festschrift für Marcel Pérennec zum 60. Geburtstag. Tübingen: 219-232.

Sandig, Barbara (1987): "Kontextualisierungshinweise: Verwendungen von so im Prozeß sprachlichen Handelns". In: Rosengren, Inger (ed.): Sprache und Pragmatik. Lunder Symposium 1986. Stockholm: 327-333.

Schanen, François/Confais, Jean-Paul (1989): Grammaire de l'allemand. Formes et fonctions. Paris.

Svorou, Soteria (1994): The Grammar of Space. Amsterdam.

Thurmair, Maria (2001): Vergleiche und Vergleichen. Eine Studie zu Form und Funktion der Vergleichsstrukturen im Deutschen. Tübingen.

Wallace, Stephen (1982): "Figure and Ground: The Interrelationship of Linguistic Categories". In: Hopper, Paul J. (ed.): Tense - Aspect. Between Semantics and Pragmatics. Amsterdam: 201-226.

Weinrich, Harald (1993): Textgrammatik der deutschen Sprache. Mannheim.

Weydt, Harald (1979a): "Immerhin". In: Weydt, Harald (ed.): Die Partikeln der deutschen Sprache. Berlin: 335-350.

Weydt, Harald (1979b): "Partikelanalyse und Wortfeldmethode: doch, immerhin, jedenfalls, schließlich, wenigstens". In: Weydt, Harald (ed.): Die Partikeln der deutschen Sprache. Berlin: 395-416.

Weydt, Harald (1979c): "Zur Unterscheidung 'semantisch - pragmatisch', dargestellt an den Partikeln jedenfalls, immerhin und schließlich". In: Rosengren, Inger (ed.): Sprache und Pragmatik. Lunder Symposium 1978. Lund: 355-370. 
Weydt, Harald (1983): "Semantische Konvergenz. Zur Geschichte von sowieso, eh, ohnehin. Ein Beitrag zum Bedeutungswandel von Partikeln". In: Weydt, Harald (ed.): Partikeln und Interaktion. Tübingen: 172-187.

Wunderlich, Dieter (1984): "Zur Syntax der Präpositionalphrasen im Deutschen". Zeitschrift für Sprachwissenschaft 3: 65-99.

Zifonun, Gisela et al. (1997): Grammatik der deutschen Sprache. 3 Bde. Berlin.

Zifonun, Gisela. (1998): "Zur Grammatik von Subsumtion und Identität: Herr Schulte als erfahrener Lehrer". Deutsche Sprache 26: 1-17. 
particulae collectae 